mputational complexity mathematical analyses

\section{tegral equations}

mputational complexity ordinary differential equations

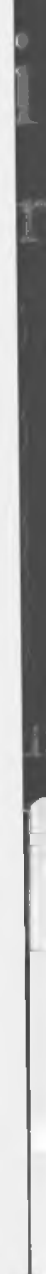

Volume 46 Number 6

ISSN 0036-1429 (print)

\section{SIAM JOURNAL ON}

\section{Numerical}

\section{Analysis}

Articles originally published online July 2008 to October 2008 ISSN 1095-7170 (electronic)

Electronic edition available at http://epubs.siam.org 


\title{
THE COMPOSITE MINI ELEMENT-COARSE MESH COMPUTATION OF STOKES FLOWS ON COMPLICATED DOMAINS*
}

\author{
DANIEL PETERSEIM ${ }^{\Downarrow}$ AND STEFAN A. SAUTER ${ }^{\dagger}$
}

\begin{abstract}
We introduce a new finite element method, the composite mini element, for the mixed discretization of the Stokes equations on two- and three-dimensional domains that may contain a hage number of geometric details. In standard finite element discretizations of the Stokes problem, such as the classical mini element, the approximation quality is determined by the maximal mesh size of the underlying triangulation, while the computational effort is determined by its number of elements. If the physical domain is very complicated, then the minimal number of simplices, which are necessary to resolve the domain, can be very large and distributed in a nonoptimal way with respect to the approximation quality. In contrast to that, the minimal dimension of the composite mini element space is independent of the number of geometric details. Instead of a geometric resolution of the domain and the boundary condition by the finite element mesh the shape of the finite element functions is adapted to the geometric details. This approach allows low-dimensional approximations even for problems with complicated geometric details such as holes or rough boundaries. We prove its linear (optimal order) approximability and its inf-sup stability. Further, we will be able to control the nonconformity in the space without increasing the space dimension in such a way that the a priori error estimate $\left\|\mathbf{u}-\mathbf{u}^{\mathrm{CME}}\right\|_{1, \Omega}+\left\|p-p^{\mathrm{CME}}\right\|_{0, \Omega} \leq h\|\mathbf{f}\|_{0, \Omega}$ holds. Thereby, in contrast to the classical methods, the choice of the mesh size parameter $h$ is not constrained by the size of geometric details. In addition, it turns out that the method can be viewed as a coarse-scale generalization of the classical mini element approach; $i_{\text {. }}$, it reduces the computational effort, while the approximation quality depends on the (coarse) mesh size in the usual way.
\end{abstract}

Key words. Finite elements, mixed methods, Stokes equation, nonconforming finite elements, space coarsening, complicated domain

AMS subject classifications. $65 \mathrm{~N} 12,65 \mathrm{~N} 15,65 \mathrm{~N} 30,65 \mathrm{D} 05,35 \mathrm{Q} 30$

DOl. $10.1137 / 070704356$

1. Introduction. The motion of a viscous incompressible fluid in a bounded, connected Lipschitz domain $\Omega \subseteq \mathbb{R}^{d}, d \in\{2,3\}$, can be described by a velocity field $\mathbf{u}: \Omega \rightarrow \mathbb{R}^{d}$ and a pressure distribution $p: \Omega \rightarrow \mathbb{R}^{d}$ which fulfill the Stokes equations under the standard Dirichlet boundary condition

$$
\left.\begin{array}{rl}
-\Delta \mathbf{u}+\nabla p & =\mathbf{f} \\
\operatorname{div} \mathbf{u} & =0
\end{array}\right\} \text { in } \Omega, \quad \mathbf{u}=\mathbf{0} \text { on } \partial \Omega
$$

where $\mathbf{f}: \Omega \rightarrow \mathbb{R}^{d}$ is a given force density. Problem (1.1) has been studied in detail, both analytically and numerically, in the literature. A wide range of finite element methods for the numerical approximation of (1.1) has been developed. In principle, these methods can also be applied to flow problems on very complicated domains $\Omega$ that arise, e.g., in environmental modeling or life sciences. However, the standard requirement that the underlying finite element mesh has to resolve the domain couples the minimal dimension of the finite element space with the number and size of geometric details, e.g., rough boundaries or holes. Such a complicated domain $\Omega$ is

- Received by the editors October 2, 2007; accepted for publication (in revised form) May 21, 2008 ; published electronically September 17, 2008.

http://www.siam org/journals/sinutr/46-6/70435.html

${ }^{\dagger}$ Institute of Mathematics, University of Zurich, Winterthurerstrasse 190, 8057 Zurich, Switzerland (dpet@math.uzh.ch, stas@math.uzh.ch). The first author's research was supported by $\$$ wiss National Science Foundation grant 200020-113457/1. 


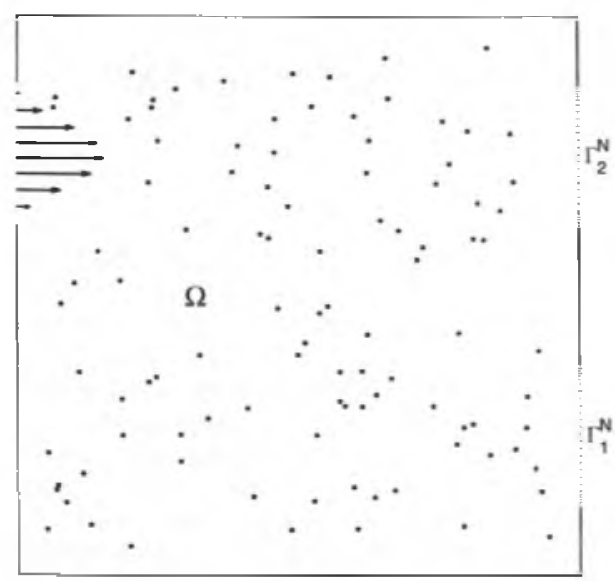

(a) Model domain $\Omega$

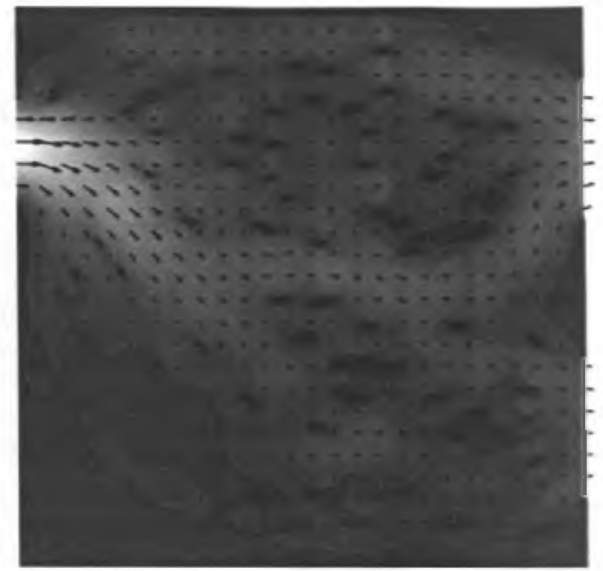

(b) Solution velocity (black $=0$, white $=$ 1).

FIG. 1.1. A model problem: Stokes flatu on the unit square with 100 randomly distributed circular holes $(r=0.005)$, an Dirichlet-inflow boundary (cf. Figure 1.3(a)), and two Neumannoutflow boundaries, $\mathrm{f}=\mathbf{0}$.

depicted in Figure 1.1(a), and a coarse triangulation of $\Omega$ is depicted in Figure 1.2(a). This triangulation is a minimal subdivision in the sense that further coarsening can be performed only by violating the shape regularity condition (cf. (2.6)). The application of standard finite elements (cf. section 2) with respect to the depicted triangulation leads to a satisfactory approximation of the corresponding Stokes flow depicted in Figure 1.1(b). However, the gain of putting a huge amount of freedom into the boundary region might be only small since the error is still mainly determined by the maximal mesh size. The use of coarser unfitted meshes is not satisfying either since the solution of the model problem behaves critically on perturbations of the model domain. as can be seen in Figure 1.3. That means that a simplification of $\Omega$ (allowing coarser subdivisions) typically will lead to poor approximations. In this paper, we will define a method that allows the low-dimensional discretization of such types of problems. while the convergence rates (with respect to the mesh size) are preserved. The main idea is to adapt the shape of the finite element functions instead of resolving the geometric details by the finite element mesh. This composite mini element belongs to the class of composite finite element methods which have been introduced to Poissontype problems and to problems in linear elasticity [12], [17]. Here we will generalize composite finite elements to the Stokes problem. Thereby the following difficulties have to be overcome:

(a) the definition of the composite mini element based on new extension operators of finite element functions for Dirichlet, Neumann, or slip-type boundary conditions,

(b) analysis: proof of convergence and discrete stability,

(c) complexity analysis.

Related approaches in the literature can be found, for instance, in [2], where unfitted meshes are used to approximate elliptic equations with Neumann boundary data. 


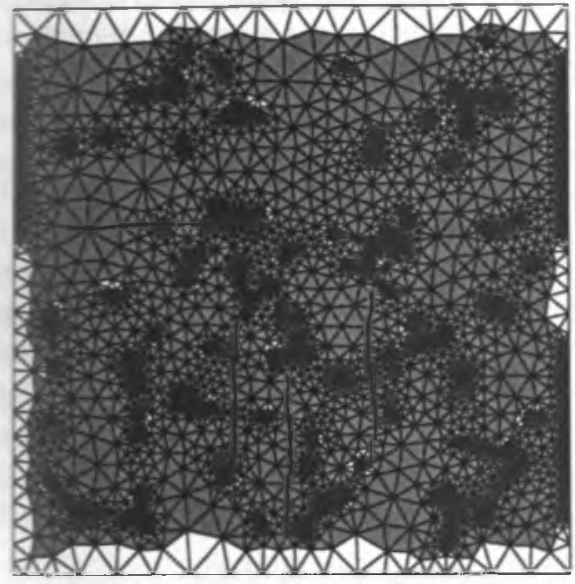

(a) Triangulation $T$ with dark-shaded inner zone $T^{\text {dof }}\left(\sharp \mathcal{T}^{\text {dof }} \approx 1000\right)$.

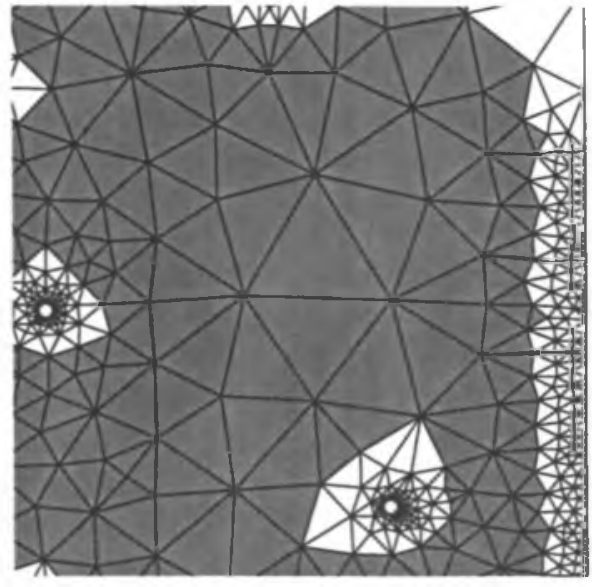

(b) $(0.8,1) \times(0.25,0.45)$-section of Figure $1.2(\mathrm{a})$.

Fig. 1.2. Shape regular triangulation $\mathcal{T}$ of the model domain $\Omega$ from Figure 1.1(a) resolving the holes as well as the in-and outflow boundaries: max. mesh size $\approx 10^{-1}$, min. mesh size $\approx 10^{-3}$, regularity constant $\approx 2$, number of triangles $\approx 9000$.

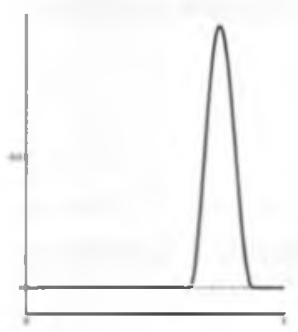

(a) Inflow velocity of the model problem.

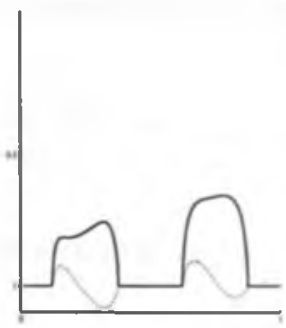

(b) Outflow velocity of the model problem.

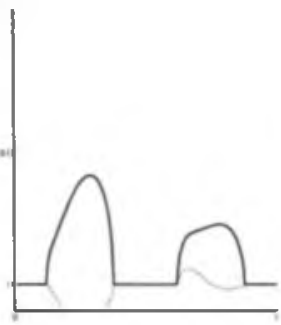

(c) Outflow velocity of the model problem with a different random hole distribution.

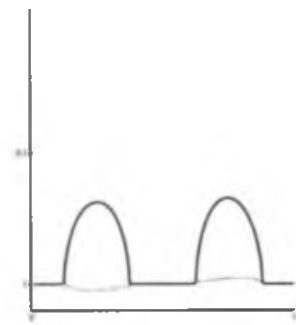

(d) Outflow velocity of the model problem without holes.

FIG. 1.3. Consequences of simplification or perturbation of the model problem introduced in Figure 1.1 on the outflow behavior: Velocity components $\left(x_{1}\right.$ solid, $x_{2}$ dotted) on $\{0\} \times(0,1)$ and on $\{1\} \times(0,1)$ for different domain modifications.

Alternative approaches in [13], [24], and [1] are not restricted to Neumann conditions. However, the coarse spaces introduced are still coupled with the geometry, and the major goal lies in the efficient solution of fine-scale discretization (see, e.g., [24] and [1]). In contrast to our approach, the asymptotic convergence order of the underlying discretization is not preserved on coarser meshes. In cases where the geometric details are distributed periodically over the domain, homogenization-based approaches used to solve Stokes problems on complicated domains provide a powerful machinery 
to construct problem-adapted finite element spaces (see, e.g., [8], [6]). In contrast, composite finite elements do not require any periodicity assumptions on the geometry.

The paper is organized as follows. In section 2 we recall the classical mini element for the weak formulation of the Stokes problem. Then, in section 3, we define the composite mini element space. Its convergence analysis is the topic of section 4 , while the final section is devoted to numerical experiments.

2. The weak Stokes problem and the classical mini element. We first introduce some basic notation. By $W_{p}^{m}(\Omega)$ we denote the Sobolev space of $\mathrm{L}^{p}$-functions with weak derivatives up to order $m \in \mathbb{N} \cup\{0\}$ in $L^{p}(\Omega), p \in \mathbb{N} \cup\{\infty\}$. In the special case $p=2$, these spaces are Hilbert spaces and are denoted by $\mathrm{H}^{m}(\Omega)$. We will write

$$
\begin{array}{lll}
\|\cdot\|_{m, p, \Omega} & \left(|\cdot|_{m, p, \Omega}\right) & \text { for the norm (seminorm) in } \mathrm{W}_{p}^{m}(\Omega), \\
\|\cdot\|_{m, \Omega} & \left(|\cdot|_{m, \Omega}\right) & \text { for the norm (seminorm) in } \mathrm{H}^{m}(\Omega), \\
\text { and }\langle\cdot, \cdot\rangle_{m, \Omega} & & \text { for the scalar product in } \mathrm{H}^{m}(\Omega) .
\end{array}
$$

We will use bold letters for the function spaces if their elements are vector valued.

The Sobolev space that contains the velocity fields of Stokes flows with a homogeneous Dirichlet boundary condition is denoted by

$$
\mathbf{H}_{0}^{1}(\Omega):=\left\{\mathbf{u} \in \mathbf{H}^{\mathbf{1}}(\Omega):\left.\mathbf{u}\right|_{\partial \Omega}=\mathbf{0} \quad \text { in the sense of traces }\right\} .
$$

The associated pressure space is $\mathrm{L}_{0}^{2}(\Omega):=\left\{p \in \mathrm{L}^{2}(\Omega): \int_{\Omega} p=0\right\}$. For a given righthand side $\mathbf{f} \in \mathbf{H}^{-1}(\Omega):=\left(\mathbf{H}_{\mathbf{0}}^{1}(\Omega)\right)^{\prime}$, the weak formulation of (1.1) reads as follow's: Find a pair $(\mathbf{u}, p) \in \mathbf{H}_{0}^{1}(\Omega) \times L_{0}^{2}(\Omega)$ such that

$$
\begin{aligned}
& \mathfrak{a}(\mathbf{u}, \mathbf{v})+\mathfrak{b}(\mathbf{v}, p)=\langle\mathbf{f}, \mathbf{v}\rangle_{0, \Omega} \quad \forall \mathbf{v} \in \mathbf{H}_{\mathbf{0}}^{1}(\Omega), \\
& \mathfrak{b}(\mathbf{u} . q) \quad=0 \quad \forall q \in \mathrm{L}_{0}^{2}(\Omega) \text {. }
\end{aligned}
$$

The bilinear forms $a: \mathbf{H}^{1}(\Omega) \times \mathbf{H}^{1}(\Omega) \rightarrow \mathbb{R}$ and $\mathbf{b}: \mathbf{H}^{1}(\Omega) \times \mathrm{L}^{2}(\Omega) \rightarrow \mathbb{R}$ are defined by

$$
\mathfrak{a}(\mathbf{u}, \mathbf{v}):=2 \int_{\Omega} \mathbf{D u}: \text { Dv. Du }:=\frac{1}{2}\left(\nabla \mathbf{u}+(\nabla \mathbf{u})^{\top}\right), \quad \mathfrak{b}(\mathbf{v}, q):=-\int_{\Omega} q \operatorname{div} \mathbf{v} .
$$

Both bilinear forms are continuous, and, due to Korn's inequality (cf. [9] and [14]). the bilinear form $\mathfrak{a}$ is coercive with respect to $\mathbf{H}_{0}^{1}(\Omega)$; i.e., there exists $a>0$ such that

$$
\mathbf{a}(\mathbf{u}, \mathbf{u}) \geq \alpha\|\mathbf{u}\|_{1, \Omega}^{2} \quad \forall \mathbf{u} \in \mathbf{H}_{\mathbf{0}}^{1}(\Omega)
$$

The coercivity constant $\alpha$ depends only on the diameter of $\Omega$. The bilinear form $b$ fulfills the inf-sup condition [10, Lemma 3.2]

$$
\inf _{0 \neq p \in \mathrm{L}_{0(\Omega)}^{2(\Omega)}} \sup _{0 \neq \mathbf{u} \in \mathbf{H}_{0(\Omega)}} \frac{b(\mathbf{u}, p)}{\|\mathbf{u}\|_{1, \Omega}\|p\|_{0, \Omega}} \geq \beta>0 .
$$

It is well known from the framework of mixed variational problems (cf. [10]) that coercivity (2.3) and stability (2.4) are sufficient conditions for the unique solvability of problem (2.1). If the solution has additional smoothness properties, i.e., $(\mathbf{u}, p) \in$ 
$\left(\mathbf{H}_{0}^{1}(\Omega) \times \mathrm{L}_{0}^{2}(\Omega)\right) \cap\left(\mathbf{H}^{2}(\Omega) \times \mathrm{H}^{1}(\Omega)\right)$, then (cf. [21]) there exists a constant $C_{\text {reg }}$ such that

$$
\|\mathbf{u}\|_{2, \Omega}+\|p\|_{1, \Omega} \leq C_{\text {reg }}\|\mathbf{f}\|_{0, \Omega} .
$$

We recall the approximation of the weak solution by the classical mini element (see, e.g., [4]). Its basis is the subdivision of the physical domain $\Omega$ into simplices, which can be performed in an exact way only if $\Omega$ is a polyhedron. In the general case, $\Omega$ needs to be approximated by a polyhedral domain $\Omega_{\mathcal{T}}$. Let $\mathcal{T}:=\left\{T_{i}: 1 \leq i \leq N\right\}$ be a subdivision of $\Omega_{\mathcal{T}}$ consisting of (closed) simplices. The mesh size of $\mathcal{T}$ is denoted by $h:=\max _{T \in T} \operatorname{diam}(T)$. Any two different simplices are supposed to either be disjoint or to share exactly either one face or one side or one vertex. We will refer to $\Theta$ as the set of vertices of $\mathcal{T}$ and to $\partial \Theta:=\Theta \cap \partial \Omega_{\mathcal{T}}$ as the set of boundary vertices. Furthermore, $\mathcal{T}$ is assumed to be shape regular; i.e., there exists $p>0$ such that

$$
\rho_{T}:=\frac{\operatorname{diam}\left(B_{T}\right)}{\operatorname{diam}(T)} \geq \rho \quad \forall T \in \mathcal{T},
$$

where $B_{T}$ denotes the largest ball contained in $T$. Based on the triangulation $\mathcal{T}$ we define the space of continuous piecewise affine functions

$$
S_{T}:=\left\{v \in C^{0}\left(\overline{\Omega_{T}}\right)|\forall T \in T: v|_{T} \in \mathbb{P}_{1}\right\} .
$$

In order to fulfill the discrete analogue of the inf-sup condition (2.4) the velocity space has to be enriched by simplex bubble functions, i.e.,

$$
B_{T}:=\operatorname{span}\left\{\psi_{T}: T \in \mathcal{T}\right\}, \quad \psi_{T}:=(d+1)^{d+1} \prod_{y \in V(T)} \lambda_{\mathrm{y}},
$$

Where $V(T)$ denotes the set of vertices of a simplex $T$ and $\lambda_{y}, y \in V(T)$, its barycentric coordinates. The unconstrained mini element space on $\mathcal{T}$ is given by

$$
\mathbf{X}_{T} \times \mathbf{M}_{T}:=\left(\mathbf{S}_{T} \oplus \mathbf{B}_{T}\right) \times S_{T},
$$

where bold letters mark vector-valued spaces. For problems with a homogeneous Dirichlet boundary condition, the mini element space is given by

$$
\mathbf{X}_{T}^{0} \times \mathbf{M}_{T}:=(\underbrace{}_{\left\{\mathbf{v} \in \mathbf{S}_{T}|\mathbf{v}|_{\partial \Omega_{T}}=0\right\}} \frac{\left.\mathbf{v} \in \mathbf{S}_{\mathcal{T}}|\mathbf{v}|_{\partial \Theta}=\mathbf{0}\right\}}{{ }_{2}} \mathbf{B}_{T}) \times S_{T} .
$$

If $\Omega=\Omega_{T}$, then the mini element space $\mathbf{X}_{T}^{0} \times \mathbf{M}_{T}$ is conforming, i.e., $\mathbf{X}_{T}^{0} \subseteq \mathbf{H}_{0}^{1}(\Omega)$. A pair $(\mathbf{u}, p) \in \mathbf{X}_{T}^{\mathbf{0}} \times\left(\mathrm{M}_{\mathcal{T}} \cap \mathrm{L}_{0}^{2}(\Omega)\right)$ is called the mini element approximation if it fulfills the discrete variational system

$$
\begin{aligned}
& \mathfrak{a}(\mathbf{u}, \mathbf{v})+\mathfrak{b}(\mathbf{v}, p)=\langle\mathbf{f}, \mathbf{v}\rangle_{0, \Omega} \quad \forall \mathbf{v} \in \mathbf{X}_{\mathcal{T}}^{0}, \\
& \mathbf{b}(\mathbf{u}, q) \quad=0 \quad \forall q \in \mathrm{M}_{T} \cap \mathrm{L}_{0}^{2}(\Omega) \text {. }
\end{aligned}
$$

It is well known that, if the continuous solution satisfies $\left(\mathbf{u}^{*}, p^{*}\right) \in \mathbf{H}^{1+r}(\Omega) \times \mathrm{H}^{r}(\Omega)$ for some $r \in\left(\frac{1}{2}, 1\right]$, the resulting method fulfills the following a priori error estimate:

$$
\left\|\mathbf{u}^{*}-\mathbf{u}\right\|_{1, \Omega}+\left\|p^{*}-p\right\|_{0, \Omega} \leq C h^{r}\|\mathbf{f}\|_{1-r, \Omega}
$$


where $C>0$ is a constant that is independent of $h, \mathbf{u}^{*}$, and $p^{*}$. As already mentioned in the introduction, estimate (2.12) indeed reflects the error but not necessarily the computational effort, namely the dimension of the finite element space. Our focus is on problems with different scales in the geometry; i.e., the domain contains parts that can be resolved by coarse meshes and (a huge number of) small geometric details. The standard (domain resolving) mini element has two disadvantages for problems of this type:

1. In practice, one is often interested in a moderate accuracy that cannot be achieved at a moderate effort if the mesh has very fine parts in order to resolve the geometry.

2. The mesh density of coarse shape regular triangulations of complicated domains is determined by the geometry and not by the smoothness properties of the solution.

The composite mini element allows a flexible handling of multiple scales in the geometry and coarse mesh computations even on very complicated domains.

3. The composite mini element. In this section we define a new mixed finite element for the Stokes equations, the composite mini element (CME). It decouples the minimal space dimension of the approximation space from the domain geometry: In the classical finite element methods this coupling is due to the fact that the boundary condition has to be incorporated into the space, which usually leads to boundary concentrated meshes. The simple idea is now not to use every vertex of the mesh as a degree of freedom, as is usually done, but to assign degrees of freedom only to nodal points at a proper distance to the boundary and "smear" the shape functions to the boundary by taking the Dirichlet condition into account. To increase flexibility we will use an overlapping triangulation $\mathcal{T}$, i.e., the condition ${ }^{1}$

$$
\Omega \subseteq \Omega_{\tau}:=\operatorname{int}\left(\bigcup_{\tau \in \mathcal{T}} \tau\right)
$$

We define shape functions depending only on a subset of nodes of $\mathcal{T}$; the remaining nodes are slave nodes. The latter are used to adapt the shape of the CME functions to the complicated zero boundary. We split the triangulation into two disjoint submeshes, a coarse (inner) part $\mathcal{T}^{\text {dof }}$ and a possibly refined (boundary) part $\mathcal{T}^{\text {slave }}$ (see Figure 1.2):

$$
\mathcal{T}=\mathcal{T}^{\text {dof }} \cup \mathcal{T}^{\text {slave }}, \quad \emptyset \neq \Omega_{T_{\text {suf }}}:=\bigcup_{T \in \mathcal{T}_{\text {dor }}} T \subseteq \Omega
$$

Accordingly, the set of vertices $\Theta$ is decomposed into $\Theta=\Theta^{\text {dof }} \cup \Theta^{\text {slave }}$, where $\Theta^{\text {dof }}$ is the set of vertices of $\mathcal{T}^{\text {dof }}$ and $\Theta^{\text {slave }}:=\Theta \backslash \Theta^{\text {dof }}$. The common vertices of $\mathcal{T}^{\text {dof }}$ and $\mathcal{T}^{\text {slave }}$ have been put into $\Theta^{\text {dof }}$, and the degrees of freedom will be associated with $\Theta^{\text {dof }}$. We refer to the vertices in $\Theta^{\text {slave }}$ as slave nodes since later the values therein are defined via extension from the inner degrees of freedom toward the boundary conditions. This approach reduces the number of unknowns in the finite element discretization of the problem to the order $\sharp \mathcal{T}^{\text {dof }}$ compared to $\sharp \mathcal{T}$. Thus, the local mesh refinements located in the slave part of the mesh do not affect the space dimension. This technique will turn out to be very efficient for problems on domains with rough outer boundaries

\footnotetext{
${ }^{1}$ This inclusion condition can be relaxed in the sense that triangulations are allowed if the errors arising from the domain approximation are negligible.
} 


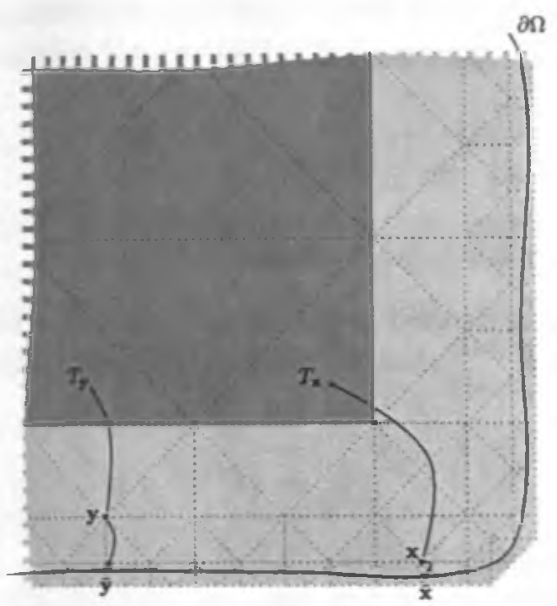

(a) The choice of closest boundary point and closest inner simplex.

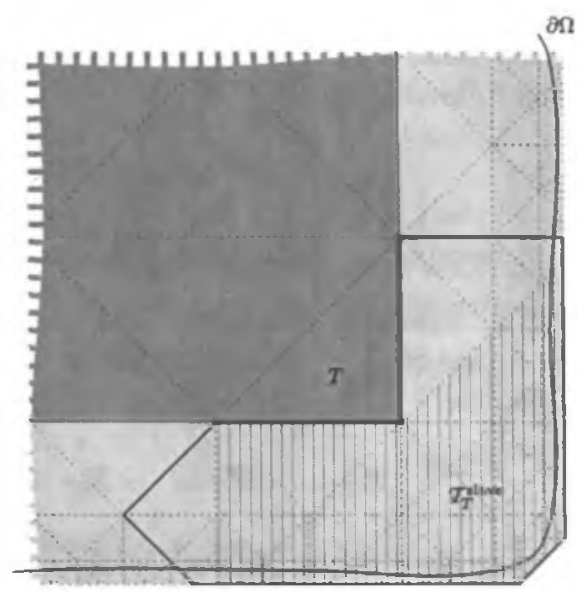

(b) Inner simplex $T$ with its domain of

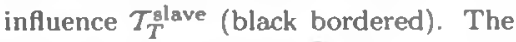
hatched subset depicts $\bar{T}_{T}^{\text {slave }}$

FIG. 3.1. Iltustration of a part of a composite mesh $\mathcal{T}$ with dark-shaded inner part $\mathcal{T}^{\text {dof }}$.

and (or) a moderate number of holes. The crucial point in the definition of the CME space is the choice of suitable extension operators for the pressure and for the velocity.

3.1. Construction of extension operators for finite elements. First, we assign a closest boundary point $\overline{\mathbf{x}} \in \partial \Omega$ and a closest inner simplex $T_{\mathbf{x}} \in \mathcal{T}^{\text {dof }}$ to every slave node $x \in \Theta^{\text {slave }}$ by

$$
\mathbf{x} \mapsto \overline{\mathbf{x}} \in \arg \inf _{\mathbf{y} \in \partial \Omega} \operatorname{dist}(\mathbf{x}, \mathbf{y}), \quad \mathbf{x} \mapsto T_{\mathbf{x}} \in \arg \min _{T \in \mathcal{T}^{\text {duof }}} \operatorname{dist}(\mathbf{x}, T) .
$$

Both mappings are illustrated in Figure 3.1. They can easily be computed in a preprocessing step, for instance, during the assembling of the triangulation (cf. [11], [16]).

3.1.1. Pressure extension. Pressure test functions $q \in S_{T_{\text {llor }} \text {, defined on the }}$ inner mesh $\mathcal{T}^{\text {dof }}$, are extended to $\Omega_{\mathcal{T}}$ by

$$
\mathcal{E}^{\mathrm{p}}: S_{\mathcal{T}_{\text {duf }}} \rightarrow S_{\mathcal{T}}, \quad\left(\mathcal{E}^{\mathrm{p}} q\right)(\mathbf{x}):= \begin{cases}q(\mathbf{x}), & \mathbf{x} \in \Theta^{\text {dof }}, \\ q_{T_{\mathbf{x}}}(\mathbf{x}), & \mathbf{x} \in \Theta^{\text {slave }},\end{cases}
$$

where $q_{T}$ denotes the affine extension (extension by itself) of $\left.q\right|_{T}$ to $\mathbb{R}^{d}$. The operator $\mathcal{E}^{\mathrm{P}}$ is well defined since its image is supposed to be a subspace of $S_{\mathcal{T}}$.

3.1.2. Velocity extension. We will use a modification of $\mathcal{E}^{\mathrm{P}}$ for the extension of the velocity which takes into account the homogeneous Dirichlet boundary condition (cf. [17])

$$
\mathcal{E}^{\mathbf{0}}: \mathbf{S}_{T^{\text {duf }}} \rightarrow \mathbf{S}_{\tau}, \quad \mathcal{E}^{\mathbf{0}} \mathbf{u}(\mathbf{x}):= \begin{cases}\mathbf{u}(\mathbf{x}) & \forall \mathbf{x} \in \Theta^{\text {dof }} \\ \mathbf{u}_{T_{\mathbf{x}}}(\mathbf{x})-\mathbf{u}_{T_{\mathbf{x}}}(\overline{\mathbf{x}}) & \forall \mathbf{x} \in \Theta^{\text {slave }}\end{cases}
$$


Note that $\left(\mathcal{E}^{\mathbf{0}} \mathbf{u}\right)(\mathbf{x})=0$ if $\mathbf{x} \in \partial \Omega$ and that only the linear part of a mini element velocity field is extended. Using the mean value theorem, $\left(\mathcal{E}^{0} \mathbf{u}\right)(\mathbf{x})$ can be rewritten in the following form:

$$
\left(\mathcal{E}^{0} \mathbf{u}\right)(\mathbf{x})=\left(\nabla \mathbf{u}_{T_{\mathbf{x}}}\right)(\mathbf{x}-\overline{\mathbf{x}}) \quad \forall \mathbf{x} \in \Theta^{\text {slave }},
$$

where $\mathbf{u}=\left(u^{1}, \ldots, u^{d}\right)^{T}$ and $\nabla \mathbf{u}_{T_{\mathbf{x}}}$ is the (constant) Jacobian matrix of $\mathbf{u}$ on $T_{\mathbf{x}}$.

3.2. Space definition. The CME space is defined as the image of the mini element space on $\mathcal{T}^{\text {dof }}$ under the linear mapping $\mathcal{E}^{\mathrm{CME}}$ that is composed of the previous extensions:

$$
\mathcal{E}^{\mathrm{CME}}: \mathbf{X}_{T_{\text {ditut }}} \times \mathrm{M}_{\mathcal{T}^{\mathrm{t} \text { in, }}} \rightarrow \mathbf{X}_{T} \times \mathrm{M}_{\mathcal{T}}, \quad\left(\mathbf{u}^{S}+\mathbf{u}^{B}, p\right) \mapsto\left(\mathcal{E}^{\mathbf{0}} \mathbf{u}^{S}+\mathbf{u}^{B}, \mathcal{E}^{\mathrm{p}} p\right)
$$

Thereby, the bubble part $\mathbf{u}^{B} \in \mathbf{B}_{\mathcal{T} \text { dof }}$ of a velocity field $\mathbf{u}=\mathbf{u}^{S}+\mathbf{u}^{B}, \mathbf{u}^{S} \in \mathbf{S}_{\mathcal{T} \text { lu }}$, is simply extended by zero. The operator $\mathcal{E}^{\mathrm{CME}}$ is injective, since functions are not changed on $\mathcal{T}^{\text {dof }}$. If any two elements of $\mathbf{X}_{\mathcal{T} \text { dut }} \times \mathrm{M}_{\mathcal{T} \text { inf }}$ are different, so are their images. Finally, the CME space is defined by

$$
\mathbf{X}_{\mathcal{T}}^{\mathrm{CME}} \times \mathrm{M}_{T}^{\mathrm{CME}}:=\mathcal{E}^{\mathrm{CME}}\left(\mathbf{X}_{T^{\text {deof }}} \times \mathrm{M}_{\mathcal{T}^{\text {(liof }}}\right) \subseteq \mathbf{X}_{\mathcal{T}} \times \mathrm{M}_{\mathcal{T}}
$$

Note that, in general, the CME is nonconforming because the Dirichlet boundary condition is satisfied only in an approximate way. This nonconformity can be controlled in an a priori or, respectively, in an a posteriori way by the local mesh size in the near boundary zone $\mathcal{T}^{\text {slave }}$. Note that there is no nonconformity arising from the pressure part of the space. A pair $(\mathbf{u}, p) \in \mathbf{X}_{T}^{\mathrm{CME}} \times\left(\mathrm{M}_{T}^{\mathrm{CME}} \cap \mathrm{L}_{0}^{2}(\Omega)\right)$ defines the CME approximation if it fulfills the discrete variational system

$$
\begin{aligned}
\mathbf{a}(\mathbf{u}, \mathbf{v})+\mathbf{b}(\mathbf{v}, p) & =\langle\mathbf{f}, \mathbf{v}\rangle_{0, \Omega} & & \forall \mathbf{v} \in \mathbf{X}_{T}^{\mathrm{CME}}, \\
\mathbf{b}(\mathbf{u}, q) & =0 & & \forall q \in \mathrm{M}_{T}^{\mathrm{CME}} \cap \mathrm{L}_{0}^{2}(\Omega) .
\end{aligned}
$$

4. A priori error analysis. In this section we will present the main theorem on the convergence of the CME method. Our results are based on the general mixed theory presented in [10] and [4]. The main steps are the investigation of the approximation properties as well as discrete coercivity and inf-sup stability.

4.1. Approximability. First, we show that $\mathbf{H}_{0}^{1}(\Omega)$-functions can be approximated by the velocity part of the CME space. Usually, a piecewise affine interpolant $\mathcal{I}_{T}$ with respect to the mesh $\mathcal{T}$ is used to prove this property. However, this is not possible in our situation because the simplices in $\mathcal{T}^{\text {slave }}$ do not contain degrees of freedom. We will use the extensions of the affine interpolants $\mathcal{I}_{\mathcal{T}}$ d., on the inner mesh $\mathcal{T}^{\text {dof }}$ instead. Therefore we may apply standard interpolation results on $\mathcal{T}^{\text {dof }}$. The approximation quality of the proposed method will depend on the choice of $\mathcal{T}^{\text {dof }}$ and the following two constants:

$$
C_{1}^{\mathcal{T}}:=\max _{t \in \mathcal{T}_{\text {tlave }}} \frac{\operatorname{dist}(t, \partial \Omega)}{\operatorname{diam}(t)} \text { and } C_{2}^{\mathcal{T}}:=\max _{\mathbf{x} \in \Theta^{\text {slave }}} \frac{\operatorname{dist}\left(\mathbf{x}, T_{\mathbf{x}}\right)}{\operatorname{diam}\left(T_{\mathbf{x}}\right)}
$$

will enter the convergence estimates. We suppose them to be moderately bounded. An algorithm for the generation of meshes such that (4.1) holds with constants of moderate size is presented in [16]. It is well known (cf. [5, Theorem 16.1]) that. for 
an arbitrary simplex $T \subseteq \mathbb{R}^{d}, d=2,3$, with regularity constant $\rho_{T}$, there exists a constant $C_{\text {int }}=C_{\text {int }}(m, p, d)$ such that

$$
\left|u-\mathcal{I}_{\mathrm{T}} u\right|_{m, \boldsymbol{p}, T} \leq \frac{C_{\mathrm{int}}}{\rho_{T}^{m}} \operatorname{diam}(T)^{\left(2-\frac{d}{2}+\frac{d}{p}-\pi r\right)}|u|_{2, I} \quad \forall u \in \mathrm{H}^{2}(T),
$$

where $m \in\{0,1\}$ and $1 \leq p \leq \infty$, provided $W_{p}^{m}(\Omega) \subseteq \mathrm{H}^{2}(\Omega) .{ }^{2} \mathcal{I}_{\mathrm{T}} u \in \mathbb{P}_{1}\left(\mathbb{R}^{d}\right)$ denotes the linear interpolant of $u$ in the vertices of $T$. The subsequent lemma analyzes the approximation quality of $\mathcal{I}_{\mathrm{T}} u$ in a neighborhood of $T$. A basic tool of the proof is an inverse estimate of the form

$$
|q|_{m, p, T} \leq\left(\frac{2}{\rho_{T}}\right)^{m} h_{T}^{\left(\frac{d}{p}-m\right)}\|q\|_{0, \infty, T} \quad \forall q \in \mathbb{P}_{1}\left(\mathbb{R}^{d}\right),
$$

where $m \in\{0,1\}$ and $p \in \mathbb{N} \cup\{\infty\}$.

LEMMA 4.1 (neighborhood property). ${ }^{3}$ Let $T$ be an arbitrary simplex with regularity constant $\rho_{T}$, and let $t$ be an arbitrary simplex with regularity constant $\rho_{t}$. Let the ratio of the diameters of $t$ and $T$ be denoted by $C_{\text {size }}$ and the distance between $T$ and $t$ relative to the size of $T$ by $C_{\text {dist }}$, i.e.,

$$
C_{\text {size }}:=\frac{\operatorname{diam}(t)}{\operatorname{diam}(T)} \text { and } C_{\text {dist }}:=\frac{\operatorname{dist}(t, T)}{\operatorname{diam}(T)} \text {. }
$$

Furthermote, let $u \in H^{2}(\operatorname{conv}(T \cup t))$, and let $\mathcal{I}_{\mathrm{T}} u \in \mathbb{P}_{1}\left(\mathbb{R}^{d}\right)$ denote the affine interpolation of $u$ at the vertices of $T$. Then, for $m \in\{0,1\}$ and $1 \leq p \leq \infty$, provided $W_{p}^{\text {mb }}(\Omega) \subseteq H^{2}(\Omega)$, there exists a constant $C_{\mathrm{np}}=C_{\mathrm{np}}\left(C_{\mathrm{int}}, d, C_{\text {size }}, C_{\mathrm{dist}}, \rho_{t}, \rho_{T}\right)>0$ such that

$$
\left|u-\mathcal{I}_{\mathrm{T}} u\right|_{m, p, t} \leq C_{\mathrm{np}} \operatorname{diam}(T)^{\left(2-\frac{d}{2}\right)} \operatorname{diam}(t)^{\left(\frac{d}{p}-m\right)}|u|_{2, \mathrm{conv}(T \cup t)} .
$$

Proof. Let $\mathcal{I}_{\mathrm{t}} u \in \mathbb{P}_{1}\left(\mathbb{R}^{d}\right)$ denote the affine interpolation of $u$ at the vertices of $t$. We define $h_{t}:=\operatorname{diam}(t)$ and $h_{T}:=\operatorname{diam}(T)$. The use of the triangle inequality, (4.2), and (4.3) leads to

$$
\begin{aligned}
u-\left.\mathcal{I}_{\mathrm{T}} u\right|_{m, p, t} & \leq\left|u-\mathcal{I}_{\mathrm{t}} u\right|_{m, p, t}+\left|\mathcal{I}_{\mathrm{t}} u-\mathcal{I}_{\mathrm{T}} u\right|_{m, p, t} \\
& \leq \frac{C_{\mathrm{int}}}{\rho_{t}^{m}} h_{t}^{\left(2 \cdots \frac{d}{2}+\frac{d}{p}-m\right)}|u|_{2, t}+\left(\frac{2}{\rho_{t}}\right)^{m} h_{i}^{\left(\frac{d}{p}-m\right)}|| \mathcal{I}_{\mathrm{t}} u-\mathcal{I}_{\mathrm{T}} u \|_{0, \infty, t} .
\end{aligned}
$$

Note that $\left\|\mathcal{I}_{\mathrm{t}} u-\mathcal{I}_{\mathrm{T}} u\right\|_{0, \infty, t}=\max _{\mathbf{x} \in V(t)}\left|\mathcal{I}_{\mathrm{t}} u(\mathbf{x})-\mathcal{I}_{\mathrm{T}} u(\mathbf{x})\right|$. We will relate the interpolates with respect to $T$ and $t$ by constructing a simplex $\tau$ that contains an arbitrary but fixed $\mathbf{x} \in V(t)$ as a vertex, while the other vertices are taken from $V(T)$. More precisely, we chose $\tau$ out of the $d+1$ simplices collected in the set

$$
\Lambda:=\{\tau \mid \tau \text { is a simplex, } \mathbf{x} \in V(\tau), V(\tau) \backslash\{\mathbf{x}\} \subseteq V(T)\} .
$$

There is at least one $\tau \in \Lambda$ (cf. Figure 4.1) that fulfills the inequality ${ }^{4}$

$$
|+\sqcap T| \geq \frac{1}{d+1}|T| \text {. }
$$

\footnotetext{
${ }^{2}$ The condition $W_{p}^{m}(\Omega) \subseteq \mathrm{H}^{2}(\Omega)$ restricts the choices of $m$ and $p$ depending on the dimension $d$. The combinations of $m$ and $p$ that will be useful later $((m, p) \in\{(0,2),(0, \infty),(1,2)\})$ are allowed in two as well as three dimensions.

${ }^{3} \mathrm{~A}$ less general two-dimensional version of this lemma has already been given in [17].

${ }^{4}$ Inequality (4.5) is sharp if $T$ is a regular simplex and $\mathbf{x}$ its midpoint.
} 


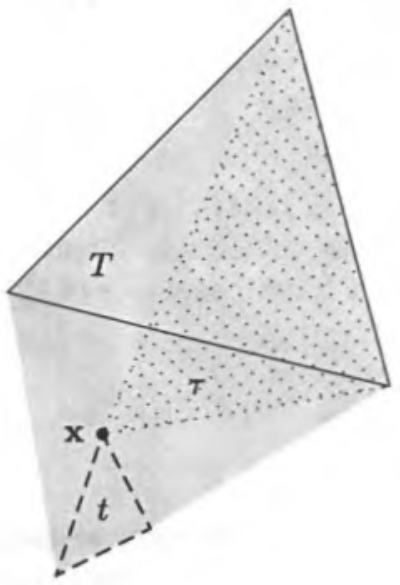

(a) $t \cap T=0 . C_{\text {dist }} \approx$ $C_{\text {size }} \approx \frac{1}{3}, t, T \subsetneq$ $\operatorname{conv}(T \cup t)$.

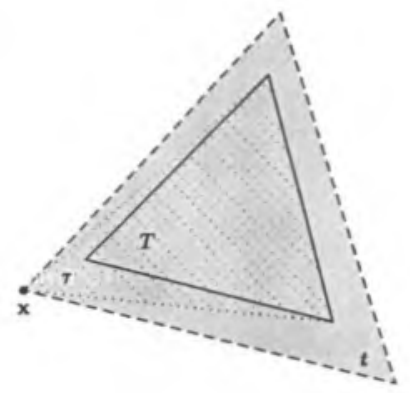

(b) $T \subseteq t, C_{\text {dist }}=0$, $C_{\text {size }} \approx \frac{3}{2}, \operatorname{conv}(T \cup t)=$ $\ell$.

FIG. 4.1. Two typical siluations for the simplices $t$ and $T$ from Lemma 4.1.

Since $\tau \cap T$ is again a simplex, we can use Heron's formula to derive

$$
\operatorname{diam}\left(B_{\tau}\right) \geq \operatorname{diam}\left(B_{\tau \cap T}\right)=\frac{d|\tau \cap T|}{|\partial(\tau \cap T)|} \geq \frac{1}{d+1} \frac{d|T|}{|\partial T|}=\frac{1}{d+1} \operatorname{diam}\left(B_{T}\right)
$$

where, again, $B_{\tau}$ (resp., $B_{\tau \cap T}$ ) is the maximal ball contained in $\tau$ (resp., $\tau \cap T$ ). For this choice of $\tau$ we get

$$
\operatorname{diam}(\tau) \leq\left(1+C_{\text {dist }}+C_{\text {size }}\right) h_{T}, \quad \rho_{\tau} \geq \frac{\rho_{T}}{(d+1)\left(1+C_{\text {dist }}+C_{\text {size }}\right)} .
$$

For $\mathbf{y} \in V(\tau) \backslash\{\mathbf{x}\}=(V(\tau) \cap V(T))$ and a Taylor expansion argument, we get

$$
\begin{aligned}
& \mid \mathcal{I}_{\mathrm{t}} u(\mathbf{x})- \mathcal{I}_{\mathrm{T}} u(\mathbf{x})|=| \mathcal{I}_{\tau} u(\mathbf{x})-\mathcal{I}_{\mathrm{T}} u(\mathbf{x}) \mid \\
&=\left|\mathcal{I}_{\tau} u(\mathbf{y})-\mathcal{I}_{\mathrm{T}} u(\mathbf{y})+\nabla\left(\mathcal{I}_{\tau} u-\mathcal{I}_{\mathrm{T}} u\right) \cdot(\mathbf{x}-\mathbf{y})\right| \\
& \leq\left|\nabla\left(\mathcal{I}_{\tau} u-\mathcal{I}_{\mathrm{T}} u\right)\right| \operatorname{diam}(\tau) \stackrel{(4.6)}{\lesssim}\left|\nabla\left(\mathcal{I}_{\tau} u-\mathcal{I}_{\mathrm{T}} u\right)\right||T|^{\frac{1}{2}} h_{T}^{\left(1-\frac{d}{2}\right)} \\
& \stackrel{(4.6),(4.5)}{\lesssim} h_{T}^{\left(1-\frac{d}{2}\right)}\left|\mathcal{I}_{\tau} u-\mathcal{I}_{\mathrm{T}} u\right|_{1, T \cap \tau} \lesssim h_{T}^{\left(1-\frac{d}{2}\right)}\left(\left|\mathcal{I}_{\tau} u-u\right|_{1, \tau}+\left|u-\mathcal{I}_{\mathrm{T}} u\right|_{1, T}\right) \\
& \quad \stackrel{(4.2)}{\lessgtr} h_{T}^{\left(1-\frac{d}{2}\right)}\left(\operatorname{diam}(\tau)+h_{T}\right)|u|_{2, \operatorname{conv}(T \cup \tau)} \stackrel{(4.6)}{\lesssim} h_{T}^{\left(2-\frac{d}{2}\right)}|u|_{2, \operatorname{conv}(T \cup t)} .
\end{aligned}
$$

Plugging (4.7) into (4.4) finishes the proof. Devoting more effort to the estimation of the constants (see [15]) leads to

$$
C_{\mathrm{np}} \leq \frac{4(d+1)^{\frac{3}{2}} C_{\text {int }}}{\rho_{t}^{m} \rho_{T}^{\left(1+\frac{d}{2}\right)}}\left(C_{\text {size }}^{\left(2-\frac{d}{2}\right)}+\left(1+C_{\text {dist }}+C_{\text {size }}\right)^{3}\right) .
$$


Next, we will show that an arbitrary $\mathbf{H}^{2} \cap \mathbf{H}_{0}^{1}(\Omega)$-function $\mathbf{u}$, which will be fixed throughout this section, can be approximated sufficiently well by $\mathcal{E}^{0}\left(\mathcal{I}_{\mathcal{T}}\right.$ dof $\left.\mathbf{u}\right) \in$ $\mathbf{X}_{T}^{C M E}$, i.e., by the extension of the piecewise affine interpolation with respect to $\mathcal{T}^{\text {dof }}$. Since in general $\Omega \subseteq \Omega_{\tau}$, it will be useful to extend $\mathbf{u}$ to the larger domain $\Omega_{T}$. It is known that, if $\Omega$ is bounded and Lipschitz, there exists a continuous, linear extension operator $\mathcal{E}: \mathrm{H}^{k}(\Omega) \rightarrow \mathrm{H}^{k}\left(\mathbb{R}^{d}\right), k \in \mathbb{N}_{0}$, such that

$$
\forall u \in \mathrm{H}^{k}(\Omega):\left.\quad \mathfrak{E} u\right|_{\Omega}=u \text { and }\|\mathfrak{E} u\|_{H^{k}\left(\mathbb{R}^{d}\right)} \leq C_{\text {ext }}\|u\|_{\mathrm{H}^{k}(\Omega)},
$$

with a constant $C_{\text {ext }}$ depending only on $k$ and $\Omega$ (cf. [20]). It is worth noting that, for domains containing a large number of holes and a possibly rough outer boundary, there exists an extension operator with moderately small norm $C_{\text {ext }}$ under mild assumptions on the geometry. For all details, including the characterization of the class of domain geometries, we refer the reader to [18]. In the following we always identify $\mathbf{u}$ with its minimal extension $\mathfrak{E} u$ without mentioning this explicitly. For $T \in \mathcal{T}$ dof the approximation results are obvious corollaries of the classical interpolation estimate (4.2). We will concentrate on the boundary region $\mathcal{T}^{\text {slave }}$. Its elements can be grouped according to their closest inner simplices. For $T \in \mathcal{T}^{\text {dof }}$ we define the set of slave simplices by

$$
\mathcal{T}_{T}^{\text {slave }}:=\left\{t \in \mathcal{T}^{\text {slave }} \mid \exists \mathbf{x} \in V(t): T_{\mathbf{x}}=T\right\} \subseteq \mathcal{T}^{\text {slave }} .
$$

$\mathcal{T}_{T}^{\text {slave }}$ is empty for all simplices that do not intersect $\partial \Omega_{\mathcal{T} \text { dor }}$. A simplex $T \in T^{\text {dof }}$ with $\mathcal{T}_{T}^{\text {slave }} \neq \emptyset$ is called an extrapolation simplex and $\mathcal{T}_{T}^{\text {slave }}$ its mesh of influence. Additionally, we fix the subsets of $\mathcal{T}^{\text {slave }}$ which are exclusively influenced by a single element $T \in \mathcal{T}^{\text {dof }}$ by

$$
\mathcal{T}_{T}^{\text {slave }}:=\left\{t \in \mathcal{T}_{T}^{\text {slave }} \mid \forall \mathbf{x} \in V(t): T_{\mathbf{x}}=T\right\} \subseteq \mathcal{T}_{T}^{\text {slave }} .
$$

An example is depicted in Figure 3.1(b). As a first step for the $\mathbf{H}^{1}$ approximation result we prove a local $\mathbf{L}^{\infty}$-estimate. Due to the extension $\mathcal{E}^{0}$ the estimate with respect to a slave simplex $t$ cannot be local in the sense that it depends only on the $\mathbf{H}^{2}$-norm of $\mathbf{u}$ on $t$. Therefore we introduce neighborhoods of slave simplices

$$
\omega_{t}:=\bigcup_{T \in \mathcal{T}^{\text {dof }}: t \in \mathcal{T}_{T}^{\text {slave }}}\left(T \cup \underset{\tau \in \mathcal{T}_{T}^{\text {slave }}}{\tau}\right), \quad t \in \mathcal{T}^{\text {slave }},
$$

and neighborhoods of extrapolation simplices

$$
\omega_{T}:=\bigcup_{t \in \mathcal{T}_{i}^{\text {Ilnve }}} \omega_{t}, \quad T \in \mathcal{T}^{\text {dof }} .
$$

Lemma 4.2. There is a constant $C=C\left(C_{\mathrm{int}}, \rho, C_{1}^{T}, C_{2}^{T}, d\right)>0$ that does not depend on $\mathbf{u}$ such that

$$
\left\|\mathbf{u}-\mathcal{E}^{0} \mathcal{I}_{\mathcal{T}_{\text {dut }} \mathbf{u}}\right\|_{0, \infty, t} \leq C\left[\left(\operatorname{diam}(t)^{(d-1)} h\right)^{\frac{1}{d}}\right]^{\left(2-\frac{d}{2}\right)}|\mathbf{u}|_{2, \omega_{T}} \quad \forall t \in \mathcal{T}^{\text {slave }}
$$

Proof. For $t \in \mathcal{T}^{\text {slave }}$ let $h_{t}:=\operatorname{diam}(t)$ and $\mathcal{I}_{\mathbf{t}} \mathbf{u}$ denote the affine interpolant of $\mathbf{u}$ at the vertices of $t$. Then

$$
\left\|\mathbf{u}-\mathcal{E}^{0} \mathcal{I}_{\mathcal{T}^{\text {dot }} \mathbf{u}}\right\|_{\infty, t} \leq\left\|\mathbf{u}-\mathcal{I}_{\mathrm{t}} \mathbf{u}\right\|_{\infty, t}+\left\|\mathcal{I}_{\mathrm{t}} \mathbf{u}-\mathcal{E}^{0} \mathcal{I}_{\mathcal{T}^{\text {dof }}} \mathbf{u}\right\|_{\infty, t}
$$




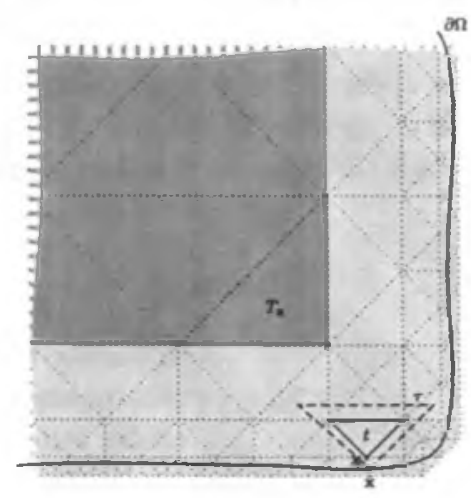

(a) Illustration of a slave node $x$ and the simplex $\tau=\tau_{x}$ from the proof of Lemma 4.2 in $\mathbb{R}^{2}$.

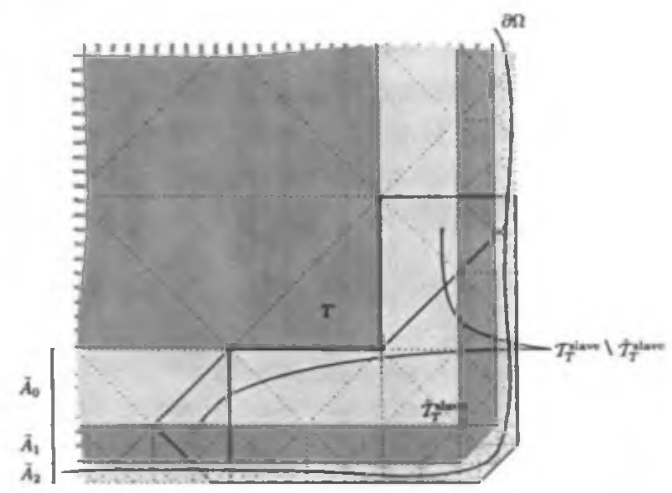

(b) The boundary regions $\bar{A}_{i}$.

FIG. 4.2. Some illustrations for the proofs of Lemma 4.2 and Theorem 4.4.

A bound of the first term in (4.9) is given by (4.2). It remains to bound the second term in (4.9) or, equivalently, $\left|\mathbf{u}(\mathbf{x})-\mathcal{E}^{0} \mathcal{I}_{\mathcal{T} \text { dor }} \mathbf{u}(\mathbf{x})\right|$ for $\mathbf{x} \in V(t)$. We construct a simplex $\tau$ (that does not necessarily belong to $T$ (see also Figure 4.2(a))) with the following properties:

1. $\mathbf{x} \in \tau$;

2. $\overline{\mathbf{x}}$ is a vertex of $\tau$;

3. $h_{\tau}:=\operatorname{diam}(\tau) \in \mathcal{O}\left(h_{t}\left(\frac{h}{h_{t}}\right)^{\frac{1}{\tau}}\right), \rho_{\tau} \geq \rho$, and $\tau \subseteq \omega_{t}$.

Due to (2.6) and (4.1) such a simplex always exists. The choice $h_{\tau} \approx h_{t}\left(\frac{h}{h}\right)^{\frac{1}{d}}$ is made in order to minimize the bound of the pointwise error (see (4.11) in this proof). Let the closest inner simplex according to $\mathbf{x}$ be denoted by $T_{\mathbf{x}}$, and let its diameter be denoted by $h_{T_{\mathbf{x}}}:=\operatorname{diam}\left(T_{\mathbf{x}}\right)$. Let $\mathcal{I}_{\tau}$ and $\mathcal{I}_{\mathrm{T}_{\mathbf{x}}}$ denote the affine interpolation operators corresponding to $\tau$ and $T_{\mathbf{x}}$. Then we get

$$
\left|\mathcal{E}^{0} \mathcal{I}_{T^{\text {det }}} \mathbf{u}(\mathbf{x})-\mathbf{u}(\mathbf{x})\right| \stackrel{(3.3)}{=}|\nabla \mathcal{I}_{\mathrm{T}_{\mathbf{x}}} \mathbf{u} \cdot(\mathbf{x}-\overline{\mathbf{x}}) \underbrace{-\frac{=\nabla\left(\mathcal{I}_{\tau} \mathbf{u}\right) \cdot(\mathbf{x}-\mathbf{x})}{\left(\mathcal{I}_{\tau} \mathbf{u}(\mathbf{x})-\mathcal{I}_{\tau} \mathbf{u}(\overline{\mathbf{x}})\right)}+\mathcal{I}_{\tau} \mathbf{u}(\mathbf{x})}_{=0}-\mathbf{u}(\mathbf{x})|
$$

$$
\begin{aligned}
& \leq\left|\nabla\left(\mathcal{I}_{\mathrm{T}_{\mathbf{x}}} \mathbf{u}-\mathcal{I}_{\tau} \mathbf{u}\right) \cdot(\mathbf{x}-\overline{\mathbf{x}})\right|+\left|\mathbf{u}(\mathbf{x})-\mathcal{I}_{\tau} \mathbf{u}(\mathbf{x})\right| \\
& \stackrel{(4.1),(4.2)}{ } \stackrel{(4.3)}{\lesssim} h_{t}\left\|\mathcal{I}_{\mathrm{T}_{\mathbf{x}}} \mathbf{u}-\mathcal{I}_{\tau} \mathbf{u}\right\|_{1, \infty, \tau}+h_{\tau}^{\left(2-\frac{d}{2}\right)}|\mathbf{u}|_{2, \tau} \\
& \lesssim \frac{\mathcal{I}_{t}}{h_{\tau}}\left\|\mathcal{I}_{\mathrm{T}_{\mathbf{x}}} \mathbf{u}-\mathcal{I}_{\tau} \mathbf{u}\right\|_{0, \infty, \tau}+h_{\tau}^{\left(2-\frac{d}{2}\right)}|\mathbf{u}|_{2, \tau} \\
& \lesssim \frac{h_{t}}{h_{\tau}}\left(\left\|\mathcal{I}_{\mathrm{T}_{\mathbf{x}}} \mathbf{u}-\mathbf{u}\right\|_{0, \infty, \tau}+\left\|\mathbf{u}-\mathcal{I}_{\tau} \mathbf{u}\right\|_{0, \infty, \tau}\right)+h_{\tau}^{\left(2-\frac{d}{2}\right)}|\mathbf{u}|_{2, \tau} \\
& \stackrel{\text { L4.1,(4.2) }}{\lesssim}\left[\frac{h_{t} h_{T_{\mathrm{jt}}}^{\left(2-\frac{d}{2}\right)}}{h_{\tau}}+h_{t} h_{\tau}^{\left(1-\frac{d}{2}\right)}+h_{\tau}^{\left(2-\frac{d}{2}\right)}\right]|\mathbf{u}|_{2, \operatorname{conv}(T \cup \tau)} .
\end{aligned}
$$

The choice of the diameter of $\tau$ as in property 3 leads to the assertion. 
Lemma 4.3. Let $m \in\{0,1\}$. There is a constant $C=C\left(C_{\mathrm{int}}, \rho, C_{1}^{T}, C_{2}^{T}, d\right)>0$ such that

$$
\left\|\mathbf{u}-\mathcal{E}^{\mathbf{0}} \mathcal{I}_{T^{\text {dor }}} \mathbf{u}\right\|_{m, t} \leq C h^{\left(\frac{2}{a}-\frac{1}{2}\right)} \operatorname{diam}(t)^{\left(\frac{5}{2}-m-\frac{3}{2}\right)}|\mathbf{u}|_{2, \omega_{\ell}} \quad \forall t \in \mathcal{T}^{\text {slave }} .
$$

Proof. IVe separate the linear part of the error by the triangle inequality to estimate

$$
\begin{aligned}
& \left|\mathbf{u}-\mathcal{E}^{0} \mathcal{I}_{T_{\text {d d }, t} \mathbf{u}}\right|_{m, t} \leq\left|\mathcal{E}^{0} \mathcal{I}_{T^{\text {dwit }} \mathbf{u}}-\mathcal{I}_{\mathbf{t}}(\mathbf{u})\right|_{m, t}+\left|\mathcal{I}_{\mathrm{t}}(\mathbf{u})-\mathbf{u}\right|_{m, t} \\
& \stackrel{(4.3),(4.2)}{\lesssim} \operatorname{diam}(t)^{\left(\frac{d}{2}-m\right)}\left\|\mathcal{E}^{\mathbf{0}} \mathcal{I}_{\mathcal{T}^{\text {(ll, },} \mathbf{u}}-\mathcal{I}_{\mathbf{t}}(\mathbf{u})\right\|_{\infty, t}+\operatorname{diam}(t)^{(2-m)}|\mathbf{u}|_{2, t} .
\end{aligned}
$$

We apply Lemma 4.2 to estimate the first summand and obtain the assertion. The resulting constant $C$ equals $\left(\frac{2}{\rho}\right)^{m}$ times the constant of Lemma 4.2 .

The error estimates in Lemmas 4.2 and 4.3 contain positive powers of diam $(t)$. Hence, it is possible to localize the approximation error with respect to the elements of

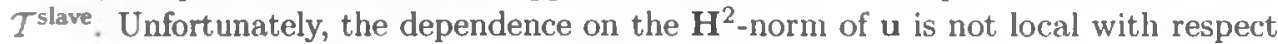
to the fine-scale mesh. Thus it is not possible to sum up the local errors to get an optimal global estimate. A deeper analysis is needed to prove the following global approximation property of the velocity space.

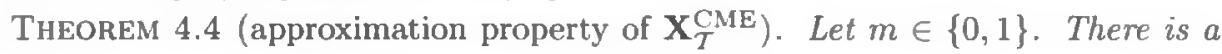
constant $C=C\left(C_{\mathrm{int}}, \rho, C_{1}^{T}, C_{2}^{T}, d, C_{\mathrm{ext}}\right)>0$ which does not depend on $h$ such that

$$
\left\|\mathbf{u}-\mathcal{E}^{0} \mathcal{I}_{T^{\text {dof }}} \mathbf{u}\right\|_{m, \Omega} \leq C h^{(2-m)}|\mathbf{u}|_{2, \Omega} .
$$

Proof. We start with a splitting of the error:

$$
\left|\mathbf{u}-\mathcal{E}^{0} \mathcal{I}_{\mathcal{T}^{\text {dot }}} \mathbf{u}\right|_{m, \Omega}^{2} \leq\left|\mathbf{u}-\mathcal{I}_{\mathcal{T}} \mathbf{u}\right|_{m, \Omega}^{2}+\sum_{T \in \mathcal{T}_{\text {dof }}} \sum_{t \in \mathcal{T}_{T}^{\text {slave }}}\left|\mathcal{I}_{\mathcal{T}} \mathbf{u}-\mathcal{E}^{0} \mathcal{I}_{\mathcal{T}^{\text {dof }}} \mathbf{u}\right|_{m, t}^{2}
$$

As indicated before, the direct application of Lemma 4.3 leads to a suboptimal error bound in the $\mathbf{H}^{1}$-case. In fact, we use Lemma 4.3 only on those (few) simplices whose nodes are assigned to different inner simplices; i.e., there does not exist a $T \in \mathcal{T}^{\text {dof }}$ such that $t \in \hat{\mathcal{T}}_{T}^{\text {slave }}$. For all other simplices we will use a more local bound. Let $T \in \mathcal{T}^{\text {dof }}$ and $t \in \hat{\mathcal{T}}_{T}^{\text {slave }}$. By $\mathcal{I}_{\mathrm{t}} \mathbf{u} \subseteq \mathbb{P}_{1}\left(\mathbb{R}^{d}\right)$ we denote the affine interpolation of $\mathbf{u}$ at the vertices of $t, h_{t}:=\operatorname{diam}(t)$. Then

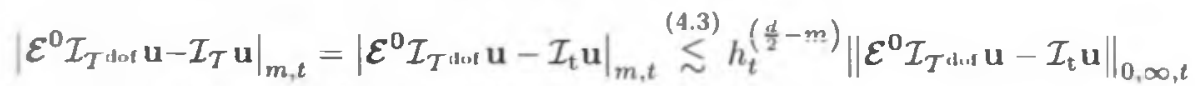

$$
\begin{aligned}
& \stackrel{(3.3)}{=} h_{t}^{\left(\frac{d}{2}-m\right)} \max _{\mathbf{x} \in V(t)}|\nabla \mathcal{I}_{\mathbf{T}_{\mathbf{x}}} \mathbf{u} \cdot(\mathbf{x}-\overline{\mathbf{x}})-\underbrace{\left(\mathcal{I}_{\mathbf{t}} \mathbf{u}(\overline{\mathbf{x}})+\nabla \mathcal{I}_{\mathbf{t}} \mathbf{u} \cdot(\mathbf{x}-\overline{\mathbf{x}})\right)}_{=\mathcal{I}_{\mathbf{t}} \mathbf{u}(\mathbf{x})}| \\
& \stackrel{(4.1)}{\lesssim} h_{t}^{\left(1+\frac{d}{2}-m\right)}\left\|\nabla\left(\mathcal{I}_{\mathrm{T}_{\mathbf{x}}} \mathbf{u}-\mathcal{I}_{\mathbf{t}} \mathbf{u}\right)\right\|_{0, \infty, t}+h_{t}^{\left(\frac{d}{2}-m\right)} \max _{\mathbf{x} \in V(t)}|\mathcal{I}_{\mathrm{t}} \mathbf{u}(\overline{\mathbf{x}})-\underbrace{\mathbf{u}(\overline{\mathbf{x}})}_{=0}|
\end{aligned}
$$

$$
\stackrel{\text { L4.1,(4.7) }}{\lesssim h_{t}^{(1-m)}}\left|\mathcal{I}_{\mathrm{T}} \mathbf{u}-\mathcal{I}_{\mathrm{t}} \mathbf{u}\right|_{1, t}+h_{t}^{(2-m)}|\mathbf{u}|_{2, \bar{t}}
$$


where $\tilde{t}:=\operatorname{conv}(t \cup\{\overline{\mathbf{x}} \mid \mathbf{x} \in V(t)\})$. We insert (4.13) into (4.12) and get

$$
\begin{aligned}
& \sum_{t \in \mathcal{T}_{T}^{\text {slave }}} \mid \mathcal{I}_{\mathcal{T}} \mathbf{u}-\mathcal{E}^{0} \mathcal{I}_{\left.\mathcal{T}_{\text {dof }} \mathbf{u}\right|_{m, t} ^{2}}
\end{aligned}
$$

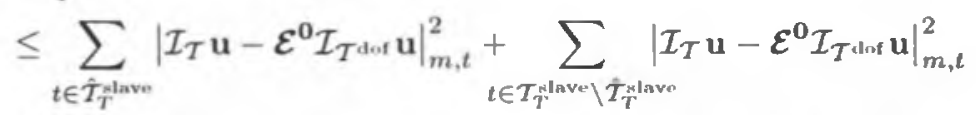

$$
\begin{aligned}
& \stackrel{\stackrel{(4.13)}{L 4.3},}{\lesssim} \sum_{t \in \hat{\mathcal{T}}_{T}^{\text {slave }}} h_{t}^{(2-2 m)}\left|\mathcal{I}_{\mathrm{T}} \mathbf{u}-\mathcal{I}_{\mathrm{t}} \mathbf{u}\right|_{1, t}^{2}+h_{t}^{(4-2 m)}|\mathbf{u}|_{2, \bar{t}}^{2}+\sum_{t \in \mathcal{T}_{T}^{\text {slave }} \backslash \hat{T}_{T}^{\text {slave }}} h^{\left(\frac{4}{d}-1\right)} h_{t}^{\left(5-2 m-\frac{4}{d}\right)}|\mathbf{u}|_{2, \omega_{t}}^{2} \\
& \leq h^{2-2 m}(\underbrace{\left|\mathcal{I}_{\mathrm{T}} \mathbf{u}-\mathcal{I}_{T} \mathbf{u}\right|_{1, \hat{\mathcal{T}}_{T}^{\text {slave }}}^{2}}_{=: M_{1, T}}+\underbrace{\sum_{t \in \hat{T}_{T}^{\text {slave }}} h_{t}^{2}|\mathbf{u}|_{2, \tilde{t}}^{2}}_{=: M_{2, T}}+\underbrace{\left.\left(\sum_{t \in \mathcal{T}_{T}^{\text {slave }} \backslash \hat{\mathcal{T}}_{T}^{\text {slave }}} h_{t}^{2}\right)|\mathbf{u}|_{2, \omega_{T}}^{2}\right)}_{=: M_{3, T}} .
\end{aligned}
$$

If we could show the existence of constants $C_{i}>0$, such that $M_{i, T} \leq C_{i} h^{2}|\mathbf{u}|_{2, \omega T}^{2}$ for all $i \in\{1,2,3\}$, then the proof is finished since

$$
\sum_{T \in T \text {.luf }}|\mathbf{u}|_{2, \omega_{T}}^{2} \leq C\left(\rho, C_{\text {ext }}\right)|\mathbf{u}|_{2, \Omega}^{2} .
$$

In case of $M_{1, T}$ this can be done in the following way:

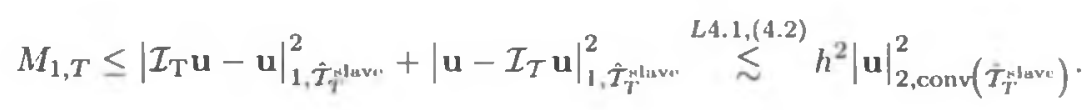

For the estimation of $M_{2, T}$ we group simplices from $\mathcal{T}^{\text {slave }}$ not only according to their extrapolation simplices but also according to their boundary distance. We define the sets

$$
A_{0}:=\mathcal{T}^{\text {slave }}, \quad A_{k}:=\left\{t \in \mathcal{T}^{\text {slave }} \mid \max _{\mathbf{x} \in t} \operatorname{dist}(\mathbf{x}, \partial \Omega)<2^{-k} h\right\}, \quad k \in \mathbb{N}
$$

and choose $K$ such that $A_{k}=\emptyset$ for all $k>K$. Their disjoint versions are given br. (cf. Figure 4.2(b))

$$
\tilde{A}_{k}:=A_{k} \backslash A_{k+1}, \quad 0 \leq k \leq K-1, \quad \tilde{A}_{K}:=A_{K} .
$$

The summation in $M_{2, T}$ can be resorted using (4.14):

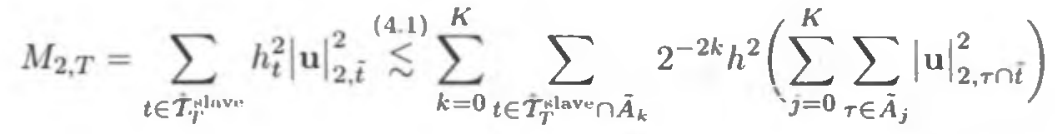

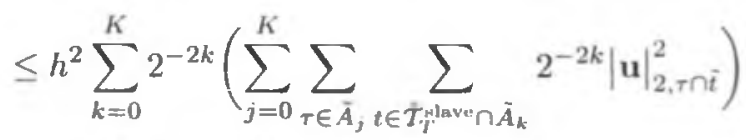

$$
\begin{aligned}
& \leq h^{2} \sum_{k=0}^{K} 2^{-2 k}\left(\sum_{j=0}^{K} \sum_{\tau \in \tilde{A}_{j}} \sharp \tilde{A}_{k, \tau}|\mathbf{u}|_{2, \tau}^{2}\right) \text {. }
\end{aligned}
$$


Here $\bar{A}_{k, \tau}:=\left\{t \in \hat{\mathcal{T}}_{T}^{\text {slave }} \cap \tilde{A}_{k} \mid \tau \cap \tilde{t} \neq \emptyset\right\}$. For $\tau \in \tilde{A}_{j}$ and $j>k, \bar{A}_{k, \tau}$ is an empty set. In the other cases, $j \leq k$, its number of elements is bounded by a constant $C\left(C_{1}^{T}, \rho, d\right)$ since

$$
\operatorname{dist}\left(t_{1}, t_{2}\right) \leq 2 \cdot 2^{-k} h \quad \forall t_{1}, t_{2} \in \tilde{A}_{k, \tau} \quad \text { and } \quad\left|t_{1}\right| \geq C\left(C_{1}^{T}, \rho\right)\left(2^{-k} h\right)^{d} \quad \forall t_{1} \in \tilde{A}_{k, \tau} .
$$

Furthermore, $\vec{A}_{k, \tau}$ is an empty set if $\tau \not L \omega_{t}$. This leads to

$$
M_{2, T} \lesssim h^{2} \sum_{k=0}^{K} 2^{-2 k} \sum_{j=0}^{k}|\mathbf{u}|_{2, \omega_{i}}^{2}=h^{2}(\underbrace{\sum_{k=0}^{K} 2^{-2 k_{k}} k}_{\leq 2})|\mathbf{u}|_{2, \omega_{T}}^{2} .
$$

It remains to estimate $M_{3, T}$. Let $N(T)$ denote the set of neighbors of $T \in T$ dof in $\mathcal{T}^{\text {dof }}$, i.e., $N(T):=\left\{\tau \in \mathcal{T}^{\text {dof }}: \bar{\tau} \cap \bar{T} \neq \emptyset\right\}$. Since $\mathcal{T}_{T}^{\text {slave }} \backslash \hat{\mathcal{T}}_{T}^{\text {slave }} \subseteq \bigcup_{\tau \in N(T)} \mathcal{T}_{T}^{\text {slave }}$ we get

$$
\begin{aligned}
M_{3, T} & \stackrel{(4.14)}{=}\left(\sum_{t \in \mathcal{T}_{T}^{\text {slave }} \backslash \mathcal{T}_{\tau}^{\text {slave }}} h_{t}^{2}\right)|\mathbf{u}|_{2, \omega_{t}}^{2} \leq\left(\sum_{k=0}^{K} \sum_{\tau \in N(T)} \sum_{t \in \mathcal{T}_{T}^{\text {slave }} \cap T_{T}^{\text {slava }} \cap \vec{A}_{k}} 2^{-2 k} h^{2}\right)|\mathbf{u}|_{2, \omega_{T}}^{2} \\
& \leq h^{2}|\mathbf{u}|_{2, \omega, T}^{2}\left(\sum_{T \in N(T)} \sum_{k=0}^{K} \#\left(\mathcal{T}_{T}^{\text {slave }} \cap \mathcal{T}_{\tau}^{\text {slave }} \cap \tilde{A}_{k}\right) 2^{-2 k}\right) .
\end{aligned}
$$

A simplex $t$ belongs to the set $\mathcal{T}_{T}^{\text {slave }} \cap \mathcal{T}_{\tau}^{\text {slave }}, \tau \in N(T)$, if it intersects the $(d-1)$ dimensional manifold on which the mapping

$$
\mathbb{R}^{d} \ni \mathbf{x} \mapsto T_{\mathbf{x}} \in \operatorname{argmin}\{\operatorname{dist}(\mathbf{x}, T), \operatorname{dist}(\mathbf{x}, \tau)\}
$$

has a jump (see also (3.1)). Therefore and due to (4.1) and shape regularity (2.6) the cardinality of $\mathcal{T}_{T}^{\text {slave }} \cap \mathcal{T}_{\tau}^{\text {slave }} \cap \tilde{A}_{k}$ satisfies

$$
\#\left(\mathcal{T}_{T}^{\text {slave }} \cap \mathcal{T}_{\tau}^{\text {slave }} \cap \tilde{A}_{k}\right) \leq C\left(C_{1}^{T}, C_{2}^{T}, p, d\right)\left(2^{k}\right)^{d-2} \quad \forall k=1, \ldots, K .
$$

In two dimensions, the bounded number of simplices belonging to $\mathcal{T}_{T}^{\text {slave }} \cap \mathcal{T}^{\top} \cap \tilde{A}_{k}$ is $\mathcal{O}$ (1). This is not true for $d=3$; however, the growth in $k$ is sufficiently slow to preserve the final estimate:

$$
M_{3 . T} \stackrel{(4.15),(4.16)}{\lesssim}\left(\sum_{k=0}^{K} 2^{-k}\right) h^{2}|\mathbf{u}|_{2, \omega_{\ell}}^{2} \leq 2 h^{2}|\mathbf{u}|_{2, \omega_{T}}^{2}
$$

The derivation of corresponding approximation results for an appropriate pressure function $p \in \mathrm{H}^{1}(\Omega)$ is less technical. However, the pointwise interpolation operator

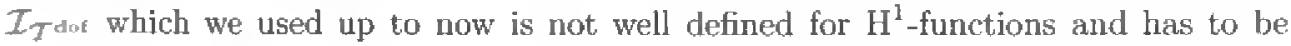
replaced by a quasi-interpolation operator $\Pi_{\mathcal{T}}: \mathrm{H}^{1}\left(\Omega_{\mathcal{T}}\right) \rightarrow S_{\mathcal{T}}$ of Scott and Zhang ${ }^{5}$ (see, e.g., [19]). Following [19, Theorem 4.1 and Corollary 4.1], the operator $\Pi_{\mathcal{T}}$ is bounded, and there exists a constant $C_{\mathrm{qint}}$ that depends only on $\rho$ such that

$$
\left\|p-\Pi_{\mathcal{T}} p\right\|_{m, t} \leq C_{\mathrm{qint}} \operatorname{diam}(t)^{1-m}\|p\|_{1, \omega_{t}} \quad \forall t \in \mathcal{T}, \quad m \in\{0.1\} .
$$

\footnotetext{
${ }^{5}$ Since we use only the approximation property of this operator and not its special structure it is also possible to use the quasi-interpolation operator as introduced by Clément [7] or its modifications as described in $[22]$ and $[23]$.
} 
As before we let $p \in \mathrm{H}^{1}(\Omega)$ be fixed and identify it with its extension $\mathfrak{E} p$ (cf. (4.8)). The error estimates with respect to the inner mesh $\mathcal{T}^{\text {dof }}$ are given in $(4.17)$, and we concentrate on the boundary part of the mesh.

THEOREM 4.5 (approximation property of $\mathrm{M}_{\mathcal{T}}^{\mathrm{CME}}$ ). There is a constant $C=$ $C\left(C_{\text {qint }}, \rho, C_{1}^{T}, C_{2}^{T}, d\right)$ such that

$$
\left\|p-\mathcal{E}^{\mathrm{p}} \Pi_{\mathcal{T}^{\text {dof }}} p\right\|_{0, t} \leq C h\|p\|_{1, \omega,} \quad \forall t \in \mathcal{T}^{\text {slave }} .
$$

Furthermore, we have the global estimate

$$
\left\|p-\mathcal{E}^{\mathrm{p}} \Pi_{\mathcal{T}^{\mathrm{d}, \mathrm{l}} \mathrm{p}} p\right\|_{0, \Omega} \leq C h\|p\|_{1, \Omega}
$$

where the constant $C$ depends only on $C_{\mathrm{ext}}, \rho$, and the constant of the local estimate.

Proof. We start by estimating the local error. Let $t \in \mathcal{T}^{\text {slave. Then }}$

$$
\begin{aligned}
& \left\|p-\mathcal{E}^{\mathrm{p}} \Pi_{\mathcal{T}^{\text {(lw, }}} p\right\|_{0, t} \leq\left\|p-\Pi_{\mathcal{T}} p\right\|_{0, t}+\left\|\Pi_{\mathcal{T}} p-\mathcal{E}^{\mathrm{P}} \Pi_{\mathcal{T} \text { du, }} p\right\|_{0, t} \\
& \text { (4.17),(4.3) }
\end{aligned}
$$

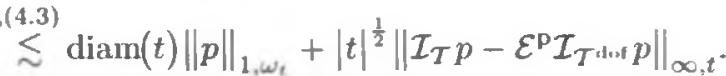

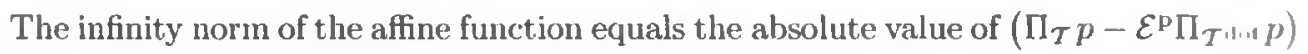
in some vertex $\mathbf{x} \in V(t)$, which can be estimated as follows:

$$
\begin{aligned}
& \left|\Pi_{\mathcal{T}} p(\mathbf{x})-\mathcal{E}^{p} \Pi_{\mathcal{T} \text { twi, }} p(\mathbf{x})\right|=\left|\Pi_{\mathcal{T}} p(\mathbf{x})-\Pi_{\mathrm{T}_{\mathbf{x}}} p(\mathbf{x})\right| \leq\left\|\Pi_{\mathcal{T}} p-\Pi_{\mathrm{T}_{\mathbf{x}}} p\right\|_{\infty, t} \\
& \lesssim|t|^{-\frac{1}{2}}\left\|\Pi_{\mathcal{T}} p-\Pi_{\mathrm{T}_{\mathrm{x}}} p\right\|_{0, t} \stackrel{L 4.1}{\lesssim} \operatorname{diam}\left(T_{\mathbf{x}}\right)|t|^{-\frac{1}{2}}\|p\|_{\mathbb{1 , L}_{i}} \cdot
\end{aligned}
$$

Hence, (4.18) is proved. The global estimate follows immediately, since the overlap of $\omega_{t}$ can be controlled in terms of $\rho$.

The preceding approximation result is one basic ingredient of the error analysis in the next section. Note that there was no restriction concerning the minimal mesh size in $\mathcal{T}^{\text {slave }}$, which will allow us to control the nonconformity in the space by adapting the local mesh size in $\mathcal{T}^{\text {slave }}$ and without increasing the space dimension. Furthermore. it is straightforward to generalize Theorems 4.4 and 4.5 to the case of less regular solutions, say $\mathbf{u} \in \mathbf{H}^{1+r}(\Omega)$ and $p \in \mathrm{H}^{r}(\Omega)$ for $r \in(0,1]$, by using the interpolation theory of Sobolev spaces (cf. [3]).

4.2. Discrete stability and coercivity. In this section, we will investigate the unique solvability of the discrete CME systems. The stability proof makes use of the boundedness of the pressure extension $\mathcal{E}^{\mathrm{p}}$.

LEMMA 4.6. There is a constant $C_{\mathcal{E}^{\mathrm{p}}}=C_{\mathcal{E}^{1}}(\rho, d)$ such that

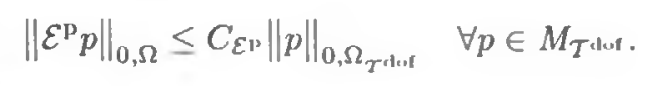

Proof. For $p \in \mathrm{M}_{\mathcal{T} \text { dor }}$ there holds

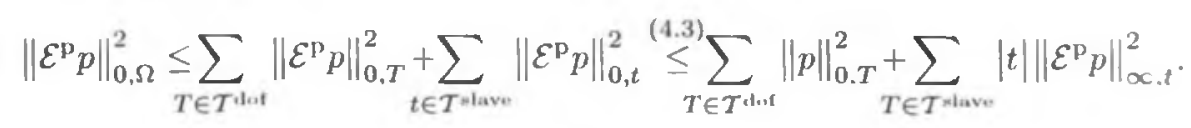

Since $\left.\mathcal{E}^{\mathrm{p}} p\right|_{t}$ takes its maximum in a vertex $\mathbf{x} \in V(t)$, there holds

$$
\left\|\mathcal{E}^{\mathrm{p}} p\right\|_{\infty, t} \leq\left\|p_{T_{\mathbf{x}}}\right\|_{\infty, t} \stackrel{L 4.1}{\leq}\left(1+\frac{\operatorname{diam}\left(T_{\mathbf{x}}\right)}{\operatorname{diam}(t)}\right)\left\|p_{T_{\mathbf{x}}}\right\|_{\infty, T_{\mathbf{x}}} \lesssim\left|T_{\mathbf{x}}\right|^{-\frac{1}{2}}\|p\|_{0, \omega_{t} \cap \Omega_{\tau^{\mathrm{dof}}}}
$$


where $p_{T_{\mathrm{x}}}$ denotes the extension of $\left.p\right|_{T_{\mathrm{x}}}$ (by itself) to $\mathbb{R}^{d}$. We plug this into (4.19), which finishes the proof, since the resulting overlap is bounded by the maximal number of neighbors in $\mathcal{T}^{\text {dof }}$ and therefore in terms of $\rho$.

Now we can show that the CMF is stable for the boundary conditions under consideration.

THEOREM 4.7 (stability), $\mathbf{X}_{T}^{\mathrm{CME}} \times M_{\mathcal{T}}^{\mathrm{CME}}$ is a stable pairing; i.e., there is a constant $\beta^{\mathrm{CME}}$ which depends only on $d, \rho$, and the discrete inf-sup constant of the mini element such that

$$
\inf _{p \in M_{T}^{\mathrm{ME}} \cap \mathbf{L}_{0}^{2}(\Omega)} \sup _{\mathbf{0} \neq \mathbf{u} \in \mathbf{X}_{T}^{\mathrm{CME}}} \frac{\mathfrak{b}(\mathbf{u}, p)}{\|\mathbf{u}\|_{1, \Omega}\|p\|_{0, \Omega}} \geq \beta^{\mathrm{CME}} .
$$

Proof. We start with the stability of the $\mathrm{CME} \mathbf{X}_{T^{\text {dof }}} \times \mathrm{M}_{\mathcal{T}_{\text {tlef }}}$ with respect to the inner mesh $\mathcal{T}^{\text {dof }}$, which is known from [4]:

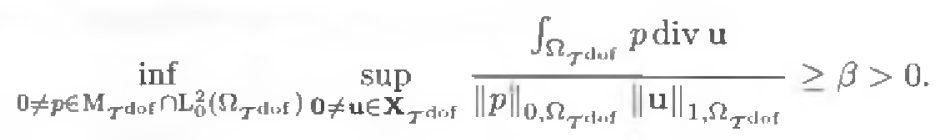

Thereby $\beta$ does not depend on the mesh size. We define two mappings to transport this result to the $\mathrm{CME}$ space. The first one is just a slight modification of $\mathcal{E}^{\mathrm{p}}$ in order to handle the $\mathrm{L}_{0}^{2}$ intersection:

$$
\overline{\mathcal{E}}^{\mathrm{p}}: \mathrm{M}_{\mathcal{T}^{\text {tho }}} \cap \mathrm{L}_{0}^{2}\left(\Omega_{\mathcal{T}^{\mathrm{d} o l}}\right) \rightarrow \mathrm{M}_{\mathcal{T}}^{\mathrm{CME}} \cap \mathrm{L}_{0}^{2}(\Omega), \quad q \mapsto \mathcal{E}^{\mathrm{p}} q-\frac{1}{|\Omega|} \int_{\Omega} \mathcal{E}^{\mathrm{p}} q
$$

Due to Lemma $4.6, \overline{\mathcal{E}}^{\mathrm{p}}$ is bounded and $\left\|\overline{\mathcal{E}}^{\mathrm{p}} q\right\|_{0, \Omega} \leq 2 C_{\mathcal{E}^{p}}\|p\|_{0, \Omega_{\tau} \text { dol }}$. Furthermore, $\tilde{\mathcal{E}}^{\mathrm{p}}$ is a bijection since $\mathcal{E}^{\mathrm{p}}$ maps constants on constants and the preimage of a constant function is constant. In a second step we construct a bounded mapping $\pi: \mathbf{X}_{T \text { dnk }} \rightarrow$ $\mathbf{X}_{\mathcal{I}}^{\mathrm{CME}}$ that satisfies

$$
\int_{\Omega} \tilde{\mathcal{E}}^{\mathrm{p}} q \operatorname{div} \pi \mathbf{u}=\int_{\Omega_{\tau^{\mathrm{dot}}}} q \operatorname{div} \mathbf{u} \quad \forall q \in \mathrm{M}_{\mathcal{T}^{\mathrm{der}}} .
$$

This step is similar to Fortin's lemma (cf. [4, Proposition II.2.9]), where such a mapping is employed to deduce the discrete stability from the continuous one. Let us suppose for the moment that $\pi$ exists. From (4.20) we know that

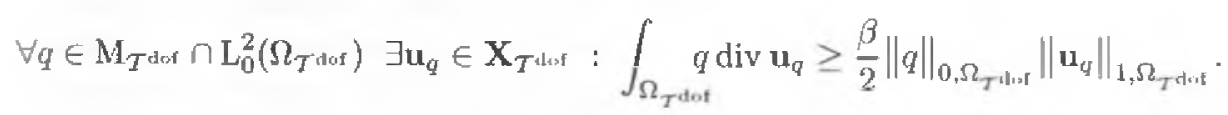

The left-hand side can be replaced using (4.21) and the bijectivity of $\tilde{\mathcal{E}}^{\text {p }}$ which leads to

$$
\begin{aligned}
& \forall p \in \mathrm{M}_{\mathcal{T}}^{\mathrm{CME}} \cap \mathrm{L}_{0}^{2}(\Omega) \exists q \in \mathrm{M}_{\mathcal{T}^{\text {dunf }}} \cap \mathrm{L}_{0}^{2}\left(\Omega_{\mathcal{T}_{\text {der }}}\right), \mathbf{u}_{q} \in \mathbf{X}_{T^{\text {duf }}}: p=\tilde{\mathcal{E}}^{\mathrm{p}} q \text { and } \\
& \int_{\Omega} p \operatorname{div} \pi \mathbf{u}_{q}=\int_{\Omega_{\tau} \text { dof }} q \operatorname{div} \mathbf{u}_{q} \geq \frac{\beta}{2}\|q\|_{0, \Omega_{T_{\text {def }}}}\left\|\mathbf{u}_{q}\right\|_{1, \Omega_{T_{\text {dof }}}} .
\end{aligned}
$$

Let $C_{\pi}$ denote the operator norm of $\pi$. Recalling $C_{\mathcal{E}}$ as in Lemma 4.6 we get

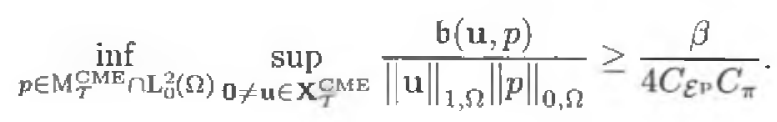




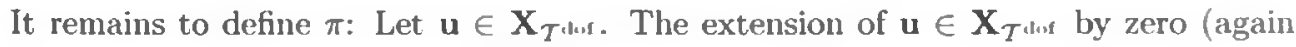
denoted by $\mathbf{u}$ ) is mapped onto $\mathbf{X}_{T}^{\mathrm{CME}}$ by using the $\mathbf{H}^{\mathbf{1}}$-orthogonal projection (denoted by $\left.\mathcal{P}_{\text {XMME }}\right)$. Due to $(4.20)$ and the coercivity of a on $\mathbf{H}_{0}^{1}\left(\Omega_{\mathcal{T}_{\text {(lir }}}\right)$ there is a unique $\mathbf{u}^{*} \in \mathbf{X}_{T_{\text {dof }}}^{\mathbf{0}}$ satisfying the following discrete Stokes problem of Dirichlet type in $\Omega_{T_{\text {iluf }}}$ :

$$
\begin{aligned}
& \int_{\Omega_{T^{\mathrm{d} o f}}} \mathrm{D}\left(\mathbf{u}^{*}\right): \mathrm{D}(\mathbf{v})-\int_{\Omega_{T^{\mathrm{d} o t}}} p \operatorname{div} \mathbf{v}=\mathbf{0} \quad \forall \mathbf{v} \in \mathbf{X}_{T^{\text {luof }}}^{\mathbf{0}},
\end{aligned}
$$

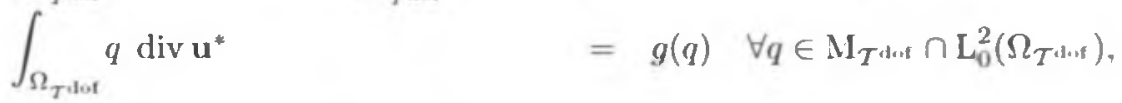

where the linear form $g: \mathrm{M}_{\mathcal{T}^{\text {dot }}} \cap \mathbf{L}_{0}^{2}\left(\Omega_{\mathcal{T}^{\text {d } w \mathrm{f}}}\right) \rightarrow \mathbb{R}$ is given by $g(q):=\int_{\Omega} \overline{\mathcal{E}}^{\mathrm{p}} q \operatorname{div}(\mathbf{u}-$ $\left.\mathcal{P}_{\mathbf{X}}^{\text {CME }} \mathbf{u}\right)$. Defining $\pi \mathbf{u}:=\mathcal{P}_{\mathbf{X}}^{\text {CME }} \mathbf{u}+\mathcal{E}^{0} \mathbf{u}^{*}$ condition (4.21) is obviously fulfilled. The operator $\pi$ is bounded because of the boundedness of the orthogonal projection and Lemma 4.6:

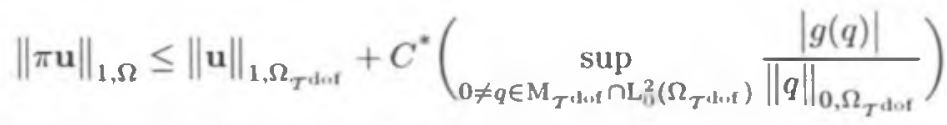

$$
\begin{aligned}
& \leq \underbrace{\left(1+C^{*}\left(1+3 C_{\mathcal{E}^{p}}\right)\right)}_{=C_{\pi}}\|\mathbf{u}\|_{1, \Omega_{T^{\mathrm{d} o f}}}
\end{aligned}
$$

It is remarkable that we deduced stability from the mini element with respect to the triangulation $\mathcal{T}^{\text {dof }}$ and not from the continuous result on the complicated domain $\Omega$.

Next, we have to investigate the coercivity of the bilinear form a with respect to the discrete space $\mathbf{X}_{T}^{\mathrm{CME}}$. We know from (2.3) that $\mathfrak{a}$ is coercive on $\mathbf{H}_{0}^{1}(\Omega)$. Since $\mathbf{X}_{T}^{\mathrm{CME}} \& \mathbf{H}_{0}^{l}(\Omega)$ this result needs to be extended to a certain neighborhood of $\mathbf{H}_{0}^{1}(\Omega)$. This neighborhood can be measured in terms of the $\mathbf{L}^{2}$-norm of the trace, as can be seen in the following lemma, the proof of which (see [15]) is analogous to the proof for the Poincaré inequality.

LEMMA 4.8 (equivalent norms in $\mathbf{H}^{1}(\Omega)$ ). For all $\mathbf{u} \in \mathbf{H}^{1}(\Omega)$ there holds $\|\mathbf{u}\|_{1, \Omega}^{2} \lesssim \mathfrak{a}(\mathbf{u}, \mathbf{u})+\|\mathbf{u}\|_{0,2 \Omega}^{2}$.

Lemma 4.8 implies that $\mathfrak{a}$ is coercive on the composite space $\mathbf{X}_{\mathcal{T}}^{\mathrm{CME}}$ if the violation of the zero boundary condition is not too large. We will make this fact precise in the subsequent lemma by showing that the nonconformity in the space can be controlled by the local mesh refinement in the slave part $\mathcal{T}^{\text {slave }}$ of the mesh $\mathcal{T}$, more precisely: by the ratio

$$
\mathrm{R}(T):=\max _{t \in \mathcal{T}_{i}^{\text {sinw": }: \text { tก } \partial \Omega \neq \emptyset}} \frac{\operatorname{diam}(t)}{\operatorname{diam}(T)}, \quad T \in \mathcal{T}^{\mathrm{dof}},
$$

which can be assigned to every extrapolation simplex.

LEMMA 4.9 (nonconformity). There is a constant $C=C\left(\rho, C_{3}^{T}\right)>0$ such that

$$
\|\mathbf{u}\|_{0, \partial \Omega} \leq C\left(\max _{T \in \mathcal{T}^{\text {lluo }}} \mathrm{R}(T)\right) h^{\frac{1}{2}}|\mathbf{u}|_{1, \Omega} \quad \forall \mathbf{u} \in \mathbf{X}_{\mathcal{T}^{\mathrm{CME}}}^{\mathrm{CM}}
$$


Proof. Let $t \in \mathcal{T}^{\text {slave }}$ such that $t \cap \partial \Omega \neq \emptyset$. We start by estimating the value of $\mathbf{u} \in \mathbf{X}_{T}^{\mathrm{CME}}$ at a vertex $\mathbf{x}$ of $t$ :

$$
|\mathbf{u}(\mathbf{x})| \stackrel{(3.4)}{\leq} \operatorname{diam}(t)\left|\nabla\left(\mathbf{u} \mid T_{x}\right)\right|=\frac{\operatorname{diam}(t)}{\left|T_{\mathbf{x}}\right|^{\frac{1}{2}}}|\mathbf{u}|_{1, T_{\mathbf{x}}}
$$

Now we can estimate the $\mathbf{L}^{2}$-norm of $\mathbf{u}$ on $\partial \Omega$ :

$$
\begin{aligned}
& \|\mathbf{u}\|_{0 . \partial \Omega}^{2} \leq \sum_{T \in T_{\text {tul }}} \sum_{t \in \mathcal{T}_{T}^{\text {slave }}: t \cap \partial \Omega \neq \emptyset}|\partial \Omega \cap t||\mathbf{u}|_{\infty \times, t}^{2}
\end{aligned}
$$

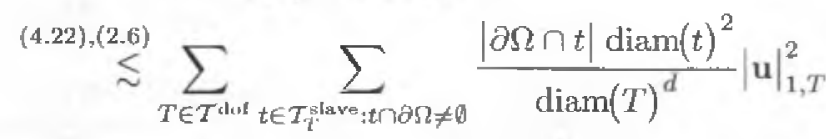

$$
\begin{aligned}
& \stackrel{(2.6)}{\lesssim} \sum_{T \in T^{\text {dot }}}\left|\partial \Omega \cap \mathcal{T}_{T}^{\text {slave }}\right| \frac{\operatorname{diam}(t)^{2}}{\operatorname{diam}(T)^{d}}|\mathbf{u}|_{1, T}^{2} \\
& \stackrel{(2.6)}{\lesssim} \underbrace{\left(\max _{T \in \mathcal{T}^{\text {dof }}} \frac{\left|\partial \Omega \cap \mathcal{T}_{T}^{\text {slave }}\right|}{\operatorname{diam}(T)^{(d-1)}}\right)}_{=: C_{3}^{T}} \frac{\operatorname{diam}(t)^{2}}{\operatorname{diam}(T)}|\mathbf{u}|_{1 . \Omega}^{2} .
\end{aligned}
$$

In Lemma 4.9 we have seen that the nonconformity in the velocity space can be controlled by the ratios $\mathrm{R}(T)$ independent from the mesh size $h$. This fact will be important for the error estimate in the subsequent section. For the unique solvability we want to avoid constraints on $\mathbf{R}(T)$. The right-hand side in Lemma 4.9 always contains at least a factor $\sqrt{h}$. So, in view of Lemma 4.8 , there is an $h_{0}$ such that the bilinear form is coercive for all triangulations with mesh size $h \leq h_{0}$. The case $h>h_{0}$ is discussed in what follows. The bilinear a has a nontrivial kernel given by the set of rigid body motions

$$
\mathcal{R}:=\left\{\mathbf{A} \cdot+\mathbf{b} \mid \mathbf{A} \in \mathbb{R}^{d \times d} \text { skew symmetric, } \mathbf{b} \in \mathbb{R}^{d}\right\},
$$

and it is therefore coercive on a subspace $\mathbf{U} \subseteq \mathbf{H}^{1}(\Omega)$ if and only if $\mathrm{U} \cap \mathcal{R}=\{0\}$. This is the key to prove discrete coercivity.

LEMMA 4.10 (discrete coercivity). There is a constant $\alpha^{\mathrm{CME}}$ that does not depend on h such that $\mathbf{a}(\mathbf{u}, \mathbf{u}) \geq \alpha^{\mathrm{CME}}\|\mathbf{u}\|_{1, \Omega}^{2}$ for all $\mathbf{u} \in \mathbf{X}_{T}^{\mathrm{CME}}$.

Proof. Let $\mathbf{u} \in \mathbf{X}_{\mathcal{T}}^{\mathrm{CME}}, \mathbf{A} \in \mathbb{R}^{d x d}$ be skew symmetric and $\mathbf{b} \in \mathbb{R}^{d}$ such that $\mathbf{u}(\mathbf{x})=\mathbf{A x}+\mathbf{b}$. Then, by definition, $\mathbf{u}(\mathbf{x})=\mathbf{A x}+\mathbf{b}=\mathbf{A}(\mathbf{x}-\overline{\mathbf{x}})$ in a slave node $\mathbf{x}$, and we get $\mathbf{A} \overline{\mathbf{x}}=-\mathbf{b}$ for all $\overline{\mathbf{x}} \in\left\{\overline{\mathbf{x}}_{i} \mid \mathbf{x}_{i} \in \Theta^{\text {slive }}\right\}$. Since $\operatorname{span}\left\{\overline{\mathbf{x}}_{i} \mid \mathbf{x}_{i} \in \Theta^{\text {slave }}\right\}=\mathbb{R}^{d}$ this can be true only if $\mathbf{A}=\mathbf{0}$ and $\mathbf{b}=\mathbf{0}$.

4.3. A priori error estimate. This section provides the main result on the convergence of the CME method.

Theorem 4.11. The discrete problem (3.6) always has a unique solution. Furthermore, if $\left(\mathbf{u}^{*}, p^{*}\right) \in\left(\mathbf{H}_{0}^{1} \cap \mathbf{H}^{1+r}(\Omega)\right) \times\left(L_{0}^{2}(\Omega) \cap H^{r}(\Omega)\right), r \in\left(\frac{1}{2}, 1\right]$, is the solution of (2.1), then we have the following a priori error estimate:

$$
\left\|\mathbf{u}^{*}-\mathbf{u}\right\|_{1, \Omega}+\left\|p^{*}-p\right\|_{0, \Omega} \leq C\left(C_{\text {appr }} h^{r}+\sup _{\substack{0 \neq \mathbf{v} \in \mathbf{X}_{T}^{\mathrm{CME}}\\}} \frac{\|\mathbf{v}\|_{0,2 \Omega}}{\|\mathbf{v}\|_{1, \Omega}}\right)\|\mathbf{f}\|_{r-1, \Omega},
$$


where the constant $C$ depends on $\alpha^{\mathrm{CME}}, \beta^{\mathrm{CME}}$, while $C_{\mathrm{appr}}$ depends on the constants in Theorems 4.4 and 4.5 .

Furthermore, if $\Omega=\Omega_{\mathcal{T}}$ or $\mathrm{R}(T) \lesssim h^{r}$ for all $T \in \mathcal{T}^{\text {dof }}$, then

$$
\left\|\mathbf{u}^{*}-\mathbf{u}\right\|_{1, \Omega}+\left\|p^{*}-p\right\|_{0, \Omega} \lesssim h^{r}\|\mathbf{f}\|_{r-1, \Omega}
$$

Proof. The unique solvability is a consequence of Theorem 4.7 and Lemma 4.10. Following the abstract mixed theory presented in [4], especially Proposition 2.16, we additionally get the following error bound:

$$
\begin{aligned}
& \left\|\mathbf{u}^{*}-\mathbf{u}\right\|_{1, \Omega}+\left\|p^{*}-p\right\|_{0, \Omega} \stackrel{T 4.10, T 4.7}{\lesssim} \inf _{\mathbf{v} \in \mathbf{X} \mathrm{CME}_{T}^{\mathrm{M}}}\left\|\mathbf{u}^{*}-\mathbf{v}\right\|_{1, \Omega}+\inf _{q \in \mathbf{M}_{T}^{\mathrm{CME}}}\left\|p^{*}-q\right\|_{0 . \Omega} \\
& +\underbrace{\sup _{0 \neq \mathbf{v} \in \mathbf{X}_{T}^{\mathrm{MME}}} \frac{\left|\mathbf{a}\left(\mathbf{u}^{*}, \mathbf{v}\right)+\mathbf{b}\left(\mathbf{v}, p^{*}\right)-\langle\mathbf{f}, \mathbf{v}\rangle_{0, \Omega}\right|}{\|\mathbf{v}\|_{1, \Omega}}}_{=: K}
\end{aligned}
$$

The infima have been estimated in section 4 . The supremum which reflects the error due to nonconformity in the space can be estimated by using the identity

$$
\int_{\Omega} \mathbf{D} \mathbf{u}^{*}: \mathbf{D v}+\int_{\Omega} p^{*} \operatorname{div} \mathbf{v}-\langle\mathbf{f}, \mathbf{v}\rangle_{0, \Omega}=\int_{\partial \Omega}\left\langle\left(\mathbf{D} \mathbf{u}^{*}-p^{*} \mathbf{I}_{\mathbf{d}}\right) \boldsymbol{\nu}, \mathbf{v}\right\rangle,
$$

which holds for the solution $\left(\mathbf{u}^{*}, p^{*}\right)$ and an arbitrary $\mathbf{v} \in \mathbf{H}^{1}(\Omega)$, and the trace theorem

$$
\left\|\mathbf{D u} \mathbf{u}^{*}\right\|_{0, \partial \Omega}^{2}+\left\|p^{*}\right\|_{0, \partial \Omega}^{2} \lesssim\left\|\mathbf{u}^{*}\right\|_{\frac{3}{2}, \Omega}^{2}+\left\|p^{*}\right\|_{\frac{1}{2}, \Omega}^{2} .
$$

Therefore the nonconformity error $K$ can be estimated as follows:

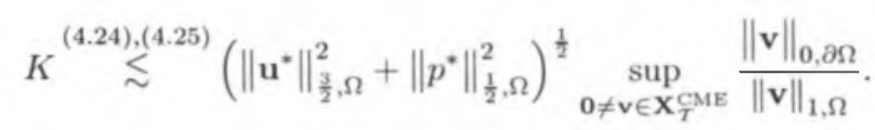

Finally, the assertion follows from the regularity estimate (2.5) and the interpolation theory of Sobolev spaces (cf. [3]).

5. Numerical experiments. Typical applications of the CME are flow problems on domains with rough outer boundaries which arise, for instance, in the modeling of rivers, lakes, and oceans where the shorelines are rarely smooth. Model problems of this type have been investigated in [15]. The results show that the theoretical estimates of the previous section are sharp for the test problems. Performance tests for composite finite elements for the Poisson equations and elasticity problems can be found in [17] and [16], where especially the use of overlapping meshes has been investigated. The subsequent experiments are addressed to flow problems on complicated domains with mixed boundary conditions. Our first model problem on the unit square with 100 randomly distributes circular holes of diameter 0.005 is depicted in Figure 1.1:

$$
\begin{aligned}
-\Delta \mathbf{u}+\nabla p & =\mathbf{0} & & \text { in } \Omega, \\
\operatorname{div} \mathbf{u} & =0 & & \text { in } \Omega, \\
\mathbf{u}(\mathbf{x}) & =0.5\left(1+\cos \left(8 \pi\left(\mathbf{x}_{2}-0.75\right)\right)\right) & & \forall \mathbf{x} \in \Gamma^{\text {in }}, \\
2(\mathbf{D u}(\mathbf{x})) \boldsymbol{\nu}(\mathbf{x}) & =p(\mathbf{x}) \boldsymbol{\nu}(\mathbf{x}) & & \forall \mathbf{x} \in \Gamma^{N}, \\
\mathbf{u}(\mathbf{x}) & =\mathbf{0} & & \forall \mathbf{x} \in \partial \Omega \backslash\left(\Gamma^{\text {in }} \cup \Gamma^{N}\right) .
\end{aligned}
$$




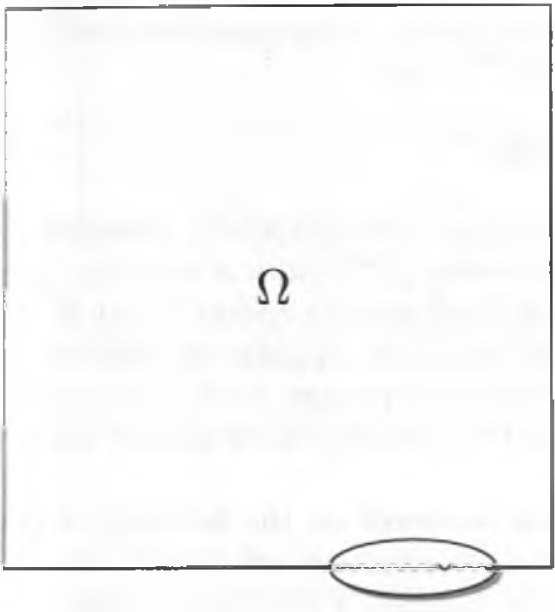

(a) Model domain $\Omega:=\left\{x \in \mathbb{R}^{2} \mid 0<\right.$ $\left.\mathbf{x}_{1}<1, g\left(\mathbf{x}_{1}\right)<\mathbf{x}_{2}<1\right\}, g\left(\mathbf{x}_{1}\right):=$ $a \sin \left(\omega \pi \mathbf{x}_{1}\right), a:=0.001, \omega:=199$.

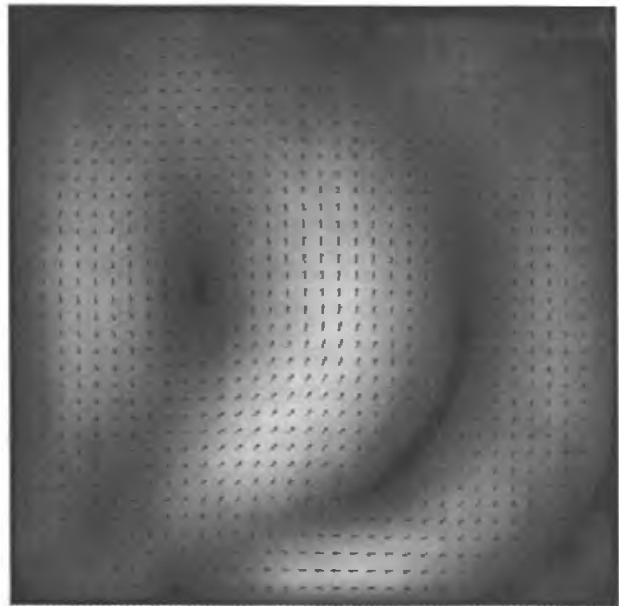

(b) Solution velocity (black $=0$, white $=$ 0.5 ).

Fic. 5.1. A model problem: Force-driven Stokes flow in a domain with a rough slip bottom boundary.

where $\Gamma^{\text {in }}:=\{0\} \times\left(\frac{5}{8}, \frac{7}{8}\right)$ and $\Gamma^{N}:=\{1\} \times\left(\frac{1}{8}, \frac{3}{8}\right) \cup\left(\frac{5}{8}, \frac{7}{8}\right)$. The difficulties of problew (5.1) not only stem from the holes but are also related to the mixed boundary conditions. The second test problem (see Figure 5.1) models a force-driven fiow in a perturbed unit square (rough bottom boundary) with mixed Dirichlet and slip boundary conditions:

$$
\begin{aligned}
-\Delta \mathbf{u}+\nabla p & =\mathbf{f} & & \text { in } \Omega, \\
\operatorname{div} \mathbf{u} & =0 & & \text { in } \Omega, \\
\mathbf{u}(\mathbf{x}) & =\mathbf{0} & & \forall \mathbf{x} \in \Gamma^{D}, \\
\langle\mathbf{u}(\mathbf{x}), \nu(\mathbf{x})\rangle & =0 & & \forall \mathbf{x} \in \partial \Omega \backslash \Gamma^{D} \\
(\mathbf{D u}(\mathbf{x})) \nu(\mathbf{x}) & =\left(\boldsymbol{\nu}(\mathbf{x})^{T}(\mathbf{D u}(\mathbf{x})) \nu(\mathbf{x})\right) \nu(\mathbf{x}) & & \forall \mathbf{x} \in \partial \Omega \backslash \Gamma^{D}
\end{aligned}
$$

where $\mathbf{f}(\mathbf{x}):=\left(\cos \left(2 \pi \mathbf{x}_{2}\right) \sin \left(2 \pi \mathbf{x}_{1}\right),\left(\frac{3}{2}-\frac{3}{2} \mathbf{x}_{2}\right)^{5} \sin \left(\pi \mathbf{x}_{2}\right) \cos \left(2 \pi \mathbf{x}_{1}\right)\right)^{T}$, and $\Gamma^{D}:=\{0\} \times$ $[0,1] \cup[0,1] \times\{1\} \cup\{1\} \times[0,1]$. Analytical solutions of (5.1) or (5.2) are not known; approximations on very fine meshes will be used for the error indication instead. We will investigate the mini element spaces with respect to coarse shape-regular meshes (see, e.g., Figures 1.2 and 5.4) for the domains of (5.1) and (5.2). They resolve the geometric details of the domain, i.e., the rough boundaries and holes, as well as the inflow and outflow boundaries. The definition of the method in section 3 has left some freedom in the choice of the inner mesh $\mathcal{T}^{\text {dor }}$ determining the CME space. We will use the simplest possible rule:

$$
t \in \mathcal{T}^{\text {dof }} \Leftrightarrow \operatorname{dist}(t, \partial \Omega)>\frac{1}{2} h^{\text {stave }} .
$$

In the case of boundary concentrated meshes as depicted in Figures 1.2 and 5.4 this choice leads to moderate constants in condition $(4.1)$, at least for $h^{\text {slave }} \in(0, h)$. Note that this choice does not take any special properties of the model problems into 
account. The sparse linear system $\mathbf{S}^{\mathrm{CME}}{ }_{\mathbf{w}} \mathrm{CME}=\mathrm{g}$, which arises from the CME discretization of (5.1) and (5.2), can be assembled locally in the usual way (see [11], [16], and [15]). The resulting system matrix is $\mathbf{S}^{\mathrm{CME}}$ fulfills

$$
\mathbf{S}^{\mathrm{CME}}=\left(\mathbf{E}^{\mathrm{CME}}\right)^{T} \mathbf{S} \mathbf{E}^{\mathrm{CME}}
$$

where $\mathbf{E}^{\mathrm{CME}}$ is the matrix representation of the linear operator $\mathcal{E}^{\mathrm{CME}}$ presented in (3.5). $\mathbf{E}^{\mathrm{CME}}$ is a sparse rectangular matrix with order $\sharp \mathcal{T}$ number of rows but only order $\sharp \mathcal{T}^{\text {dof }}$ number of columns. It is obvious that the composite system matrix $\mathrm{S}^{\mathrm{CA}} \mathrm{E}$ is (much) smaller than the classical mini element matrix $\mathbf{S}$. Sparsity can thereby be preserved since the support of the CME basis functions remains local. As a consequence the storage requirements are reduced, and a (possibly) much smaller system has to be solved.

Before we present numerical results we will comment on the handling of the boundary conditions that are not covered by the theory presented before. The inhomogeneous Dirichlet boundary condition can be handled in the standard way. By introducing a discrete vector field $\mathbf{u}_{0}: \Omega \rightarrow \mathbb{R}^{2}$ that approximates the inflow condition sufficiently well the problem can be reduced to a homogeneous problem with a modified right-hand side. Note that $\mathbf{u}_{0}$ can be chosen in the full mini element space based on a possibly fine mesh close to the inhomogeneous Dirichlet boundary which allows us to handle even complicated Dirichlet data. It is therefore independent from the choice of $h^{\text {slave }}$. From the pressure part of the CME space we know how to deal with functions that are unconstrained on the domain boundary. We simply use $\mathcal{E}^{p}$ componentwise to define the values of the velocity test functions close to the Neumann boundary. For the slip boundary condition in (5.2) we use $\mathcal{E}^{\mathrm{p}}$ in the tangential direction and $\mathcal{E}^{\mathbf{0}}$ in the normal direction. A theoretical justification of this procedure has been presented in [15], where the use of the slip boundary condition is discussed in detail. The question of which extension has to be used in a slave node $\mathbf{x}$ is decided by its closest boundary point $\overline{\mathbf{x}}$ that belongs to either the Dirichlet, Neumann, or slip boundary.

We will use problem (5.1) to investigate the behavior of the error and the system dimension on two coarse triangulations for varying choices of the parameter $h^{\text {slave }}$. Figure 5.2 summarizes the results: In both plots the lower curves ( $\square$ ) represent the compression rates, that is, the quotients between the dimensions of the composite spaces for varying values of $h^{\text {slave }}$ and the classical mini element space. The upper curves (o) show the relative errors (left: velocity, right: pressure), more precisely: the quotient of the errors of the composite approximations and the full mini element approximation. In between, the dotted curves $(x)$ are the product of compression rate and relative error. It can be seen that the errors stay almost constant as $h^{\text {slave }}$ tends to $h$. Since the space dimension rapidly decreases at the same time, the efficiency of the composite method increases. Only for values of $h^{\text {slave }}>h$ does the pressure error become large, which indicates that the pressure extension depends more critically on the choice of $h^{\text {slave }}$. This is not surprising, since information from the boundary conditions is not available. However, the velocity error in Figure 5.2(a) is not affected from the worse pressure approximation, and the behavior is fine if $h^{\text {slave }}<h$. In Figure 5.3 it can be observed that the generalization of the composite method to Neumann boundaries works well for the model problem under consideration. Due to the extension there is an expected loss in smoothness, but the general outflow behavior can be captured without placing additional degrees of freedom. This indicates a certain potential of the method not only for problems with complicated boundaries 


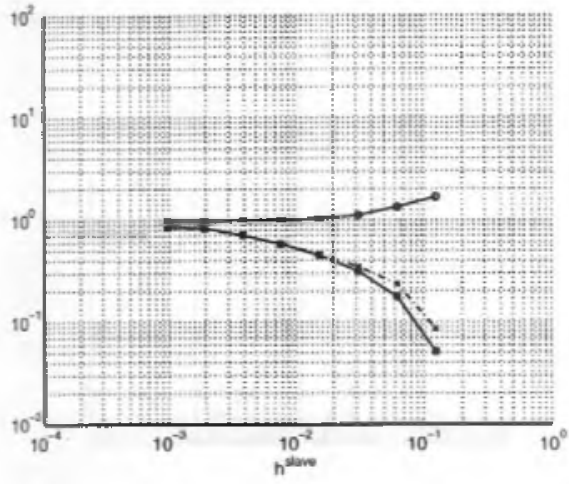

(a) Relative $\mathbf{H}^{1}(\Omega)$ velocity error (o) and relative space dimension ( $\square$ ) versus $h^{\text {slave }}, h=0.05$.

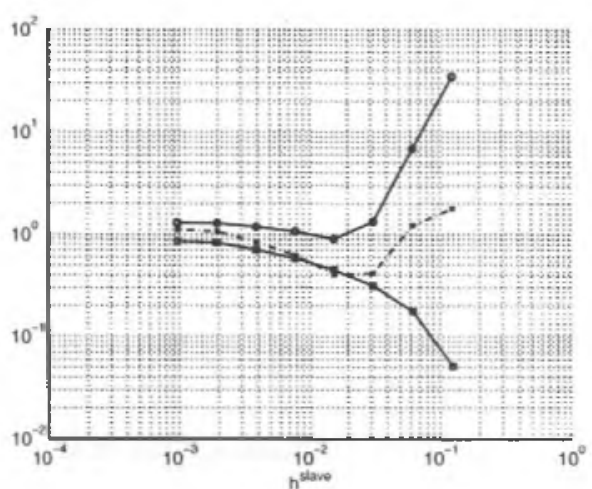

(b) Relative $\mathrm{L}^{2}(\Omega)$ pressure elror (o) and relative space dimension ( $\square$ ) versus $h^{\text {slave }}, h=0.05$.

FIG. 5.2. Relative errors $\frac{\operatorname{crror}(C M E)}{\mathrm{err}(M \mathrm{ini})}$ in suitable Sobolev norms (o) and relative system dimensions $\frac{\operatorname{dim}(C M E)}{\operatorname{dim}(M i n i)}$ (口) for resolving triangulations with $h=0.05$ under the variation of the parameler $h^{\text {slave }} \in\left(10^{-3}, 1\right)$. The product of relative error and relative dimensian, depicted as a dashed line, indicates the efficiency of the coarsening process.

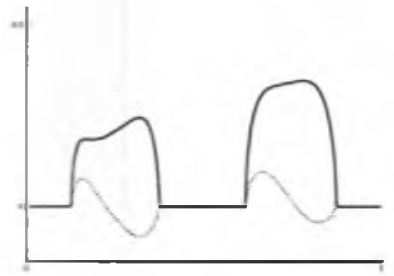

(a) Reference output.

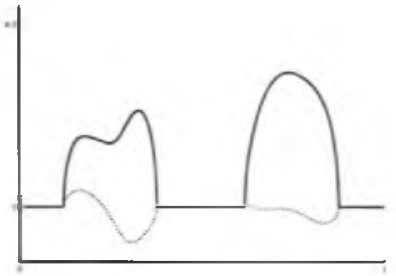

(b) Mini element output, space dimension $\approx 7$. $10^{4}$.

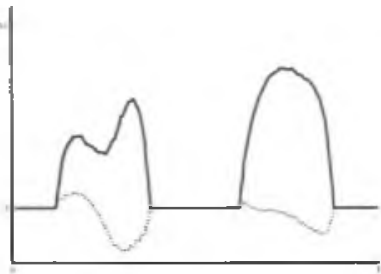

(c) CME output, space dimension $\approx 10^{4}$.

FIG. 5.3. Outflow behavior of the model problem of Figure 1.1(a): Velocity components ( $x_{1}$ solid, $x_{2}$ dotted) on $\{0\} \times(0,1)$ and on $\{1\} \times(0,1)$ for the reference approximation $(a)$, the full mini element approximation (b) on the triangulation from Figure 1.2, and composite approximation (c) for $h^{\text {slave }} \approx \frac{h}{4} \approx 0.025$.

but also with complicated boundary conditions, i.e., complicated Dirichlet data or complicated distribution of mixed Dirichlet-Neumann boundary conditions.

Finally, we use the second model problem (5.2) to investigate the convergence rates as the mesh size $h$ decreases. Thereby we compare the classical mini element on a resolving triangulation (Mini), its composite version (CME) for $h^{\text {slave }}=0.1 h$, and a classical mini element with respect to nonmatching, quasi-uniform triangulations (uniformMini). All approaches and the according parameter choices are summarized in Figure 5.4.

The results of the slip model computations are depicted in Figure 5.5, where the dependence of the velocity and pressure errors on the mesh size and the system 


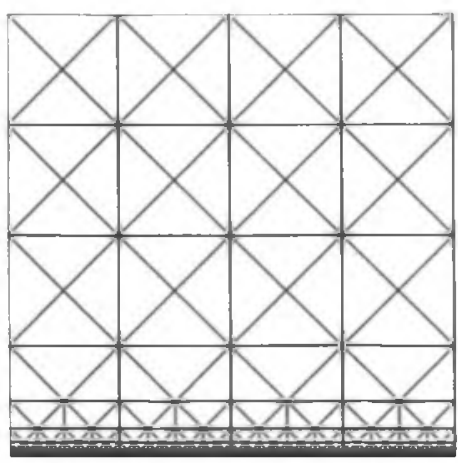

(a) Classical mini element (Mini) on a resolving mesh (minimal mesh size $2^{-10}$ ).

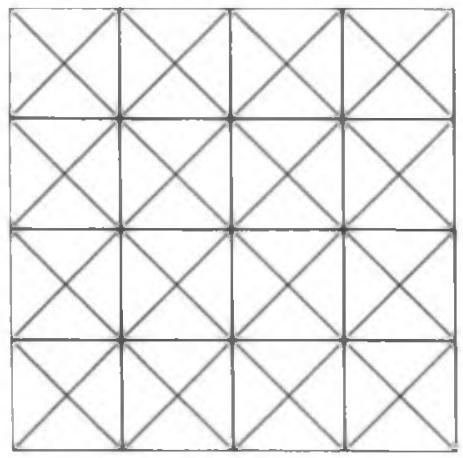

(c) Nonconforming classical mini element (uniformMini) on quasi-uniform mesh.

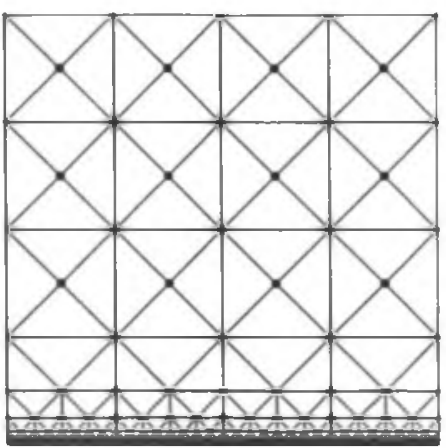

(b) Composite mini element (CME) on a resolving mesh with marked degrees of freedom (•) (minimal mesh size $\left.2^{-10}, h^{\text {slave }}=h\right)$.

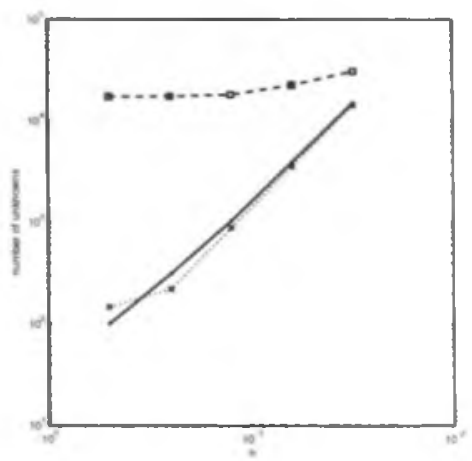

(d) System dimension as a function of $h$ : Mini (dashed), CME (solid), uniformMini (dotted).

FIG. 5.4. The grids with degrees of freedom of the different approaches for the solution of the model problem (5.2).

dimension are depicted. We make the following observations:

1. The (conforming) mini element and its composite version converge at the predicted optimal (linear) rate (cf. Figure 5.5(a)). The compressed method CME is only slightly worse than the full version (cf. Figure 5.5(a)). Note that the error of the composite method is larger than full mini error only in a near boundary zone. Away from the rough boundary the errors are almost equal: i.e., pollution effects cannot be observed for this test case (cf. Figures 5.5(b)).

2. The quasi-uniform approach is not competitive (cf. Figures $5.5(\mathrm{a})-(\mathrm{b})$ ). The reason is that the very crude approximation of the boundary conditions spoils the overall discretization too significantly. That means that our choice of minimal mesh size for the boundary resolution cannot be weakened without increasing the errors. 


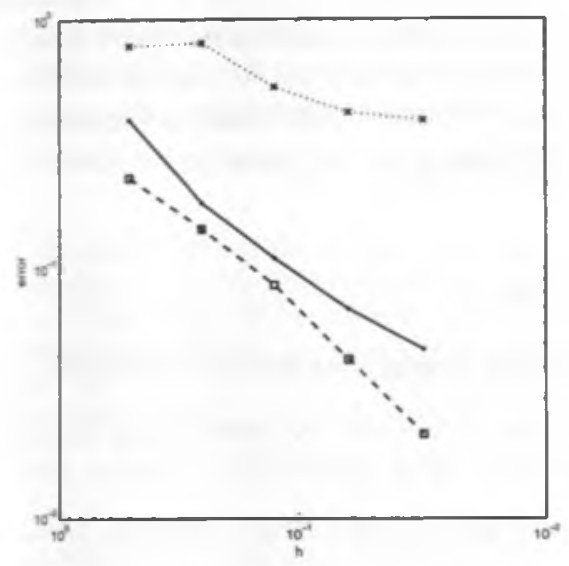

(a) Error on $\Omega$ as a function of $h$.

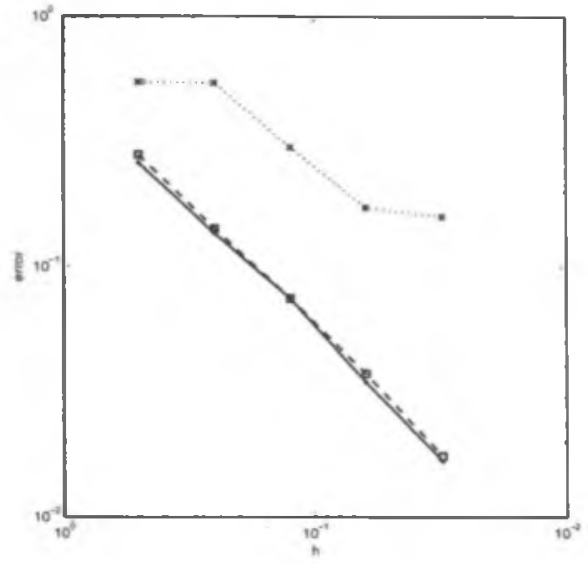

(b) Error on $(0,1) \times(0.1,1)$ as a function of $h$.

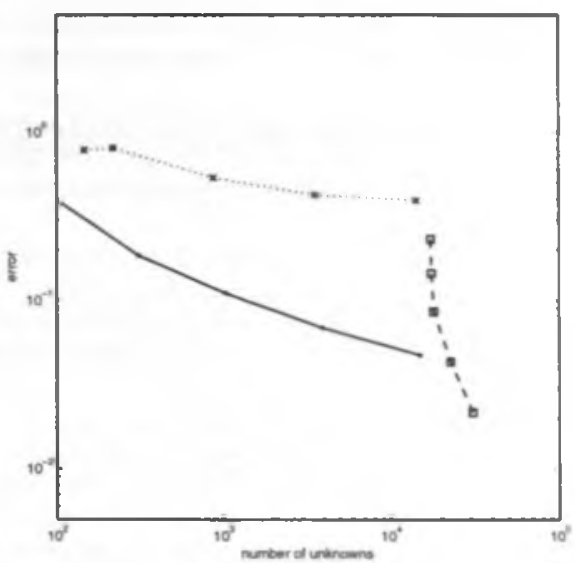

(c) Error on $\Omega$ as a function of the system dimension.

FIG. 5.5. Convergence of the methods applied to model problem (5.2). The errors (sum of $H^{1}$-velocity and $L^{2}$-pressure error) are plotted versus the maximal mesh size $h$ and the system dimension: Mini (dashed, 口), CME (solid, o), uniformMini (dotted, $x$ ).

3. The dimension of the CME space behaves like $h^{-d}$ (cf. Figure 5.4(d)). That means that the CME preserves the accuracy of the full mini element space on the coarser levels, while the cost is comparable with the quasi-uniform approach. This underpins the efficiency of our "fuzzy" treatment of the boundary conditions.

4. The composite method is the most efficient method in this test case. Though the full mini element produces slightly smaller errors than the composite element, the resulting system dimension is up to 100 times bigger than for CME on the coarsest level (see Figure 5.5(c)). In contrast to the CME the less costly quasi-uniform method produces unsatisfying approximations. 
The gain of the composite method depends on the maximal mesh size $h$. If $h$ is small enough to resolve the domain, the composite and the full method are almost equal in cost and accuracy. If one is satisfied with only moderate errors, or one is restricted to smaller dimensions, then the composite method is the better choice. Refinements in the triangulation which are due to geometric issues do not need to be treated bydegrees of freedom.

\section{REFERENCES}

[1] R. E. BANK AND J. XU, An algorithm for coarsening unstructured meshes, Numer. Math., 73 (1996), pp. 1-36.

[2] J. W. Barrett and C. M. Elliott, A finite-element method for solving elliptic equations with Neumann data on a curved boundary using unfitted meshes, IMA J. Numer. Anal.. 4 (1984), pp. 309-325.

[3] S. Brenner And L. R. Scott, The Mathematical Theory of Finite Element Methods. SpringerVerlag, New York, 1994.

[4] F. Brezzi And M. Fortin, Mixed and Hybrid Finite Element Methods, Springer-Verlag, New York, 1991.

[5] P. G. Ciarlet, The Finite Element Method for Elliptic Problems, North-Holland, Amsterdam. 1978.

[6] D. Cioranescu, P. Donato, And H. I. Ene, Homogenization of the Stokes problem with nonhomogeneous slip boundary conditions, Math. Methods Appl. Sci., 19 (1996). pp. 857-881.

[7] PH. CLÉMENT, Approrimation by finile element functions using local regularization, RAIRO Anal. Numerr., 9 (1975), pp. 77-84.

[8] A. Damlamian and P. Donato, Homogenization with small perforations of increasingly complicated shapes, SIAM J. Math. Anal., 22 (1991), pp. 639-652.

[9] G. Duvaut And J. L. Lions, Inequalities in Mechanics and Physics, Springer-Verlag. Berlin. Heidelberg. New York, 1976.

[10] V. Gikault And P.-A. Raviaht, Finite Element Approximation of the Navier-Stokes Equations, Springer-Verlag, Berlin, Heidelberg, New York, 1979.

[11] W. HackbuSCh AND S. A. SaUter, Composite finite elements for problems containing small geometric details. Part II: Implementation and numerical results, Comput. Vis. Sci.. I (1997) pp. 15-25.

[12] W. Hackbusch AND S. A. SAUter, Composite finite elements for the approximation of PDEs on domains with complicated micro-structures, Numer. Math., 75 (1997), pp. 147-472.

[13] R. Kornhuber and H. YSerentant, Multilevel methods for elliptic problems on domains not resolved by the coarse grid, in Domain Decomposition Methods in Scientific and Engineering Computing, Contemp. Math. 180, AMS, Providence, RI, 1994, pp. 49-60.

[14] J. A. NitsChe, On Korn's second inequality, RAIRO Anal. Numér., 15 (1981), pp. 237-248.

[15] D. Peterseim, The Composite Mini Element: A Mixed FEM for the Stokes Equations on Complicated Domains, Ph.D. thesis, Universität Zürich, Zürich, Switzerland. 2007.

[16] M. REch, Composite Finite Elements: An Adaptive Two-Scale Approach to the Nonconforming Approximation of Dirichlet Problems on Complicated Domains, Ph.D. thesis, Universität Zürich, Zürich, Switzerland, 2006.

[17] M. Rech, S. SAUter, ANd A. Smolianski, Two-scale composite finite element method for Dirichlet problems on complicated domains, Numer. Math., 102 (2006), pp. 681-708.

[18] S. A. SAUTER AND R. WARNKE, Extension operators and approximation on domains containing small geometric details, East-West J. Numer. Math., 7 (1999), pp. 61-77.

[19] L. R. SCOTT AND S. ZHANG, Finite element interpolation of nonsmooth functions satisfying boundary conditions, Math. Comp., 54 (1990), pp. 483-493.

[20] E. M. STEIN, Singular Integrals and Differentiablity Properties of Function, Princeton University Press, Princeton, NJ, 1970.

[21] R. TemaM, Navier-Stokes Equations, Theory and Numerical Analysis, North-Holland. Amsterdam, 1984.

[22] R. Verfürth, A Review of A Posteriori Error Estimation and Adaptive Mesh-Refinement Techniques, Teubner-Wiley, Stuttgart. 1996.

[23] R. VerfürTh, Error estimates for some quasi-interpolation operators, M2AN Math. Model. Numer. Anal., 33 (1999), pp. 695-713.

[24] H. Yserentant, Coarse grid spaces for domains with a complicated boundary. Numer. Algorithms, 21 (1999), pp. 387-392. 